\title{
Joint design of beamforming and time switching/power splitting for wireless-powered multi-antenna dual-relay network
}

\author{
Guojie Hu ${ }^{*}$, Yueming Cai, Liang Ao and Xiangdong Wang
}

\begin{abstract}
In this paper, we consider a wireless-powered dual-relay network consisting of one multi-antenna source, two single-antenna energy-constrained relays and one single-antenna destination without direct source to destination link. In order to establish the communication flow, the energy-constrained relays harvest energy from the radio frequency transmitted by the source firstly, then exploit the harvested energy to forward the source information to the destination based on distributed space time coding (DSTC). Under this network architecture, three decode-and-forward (DF) technique-based relaying protocols, i.e., time switching-based relaying (TSR) protocol, power splitting-based relaying (PSR) protocol, and hybrid relaying (HR) protocol, are considered to drive the energy transfer and information transmission. To maximize the network throughput, the joint design for the optimal energy and information beamforming vectors employed at the source, the optimal time switching, and power splitting ratios under these three protocols are investigated and solved efficiently by employing simple sequential optimization approach or alternating optimization approach. Simulations are conducted to show the superior performance achieved by our proposed scheme. Moreover, we find that the TSR protocol outperforms the PSR protocol in the low signal-to-noise ratio (SNR) region, while the latter outperforms the former in the high SNR region. And the HR protocol achieves the best performance in any SNR region. At the same time, the effect of the relays' locations on the throughput performance of these three protocols is also investigated.
\end{abstract}

Keywords: Wireless powered communication network, Beamforming vector design, TSR, PSR, HR

\section{Introduction}

With the rapid rise of the Internet of Things (IoT) [1] industry, massive intelligent devices will be deployed in anywhere to monitor engineering structures, diagnose the physical condition of the patients, report the real-time traffic information, and so on. A major problem facing is that how to prolong the life of the energy-constrained devices, so that they could be utilized in a sustainable and low-cost way. Currently, a feasible solution is to adopt the radio frequency (RF) signal-based wireless energy transfer (WET) technique [2], as RF signal is controllable, stable,

*Correspondence: Igdxhgj@sina.com

Institute of Communication Engineering, Army Engineering University of PLA, No. 2, Biaoying Street, Nanjing, China and efficient compared to the natural sources such as solar and wind.

By integrating WET and wireless data communications, wireless powered communication network (WPCN) [3-8] has raised as an emerging research topic. Up to now, the most popular research model under WPCN was the threenode relay network [9-14], in which the "harvest-thentransmit" (HTT) scheme was employed, i.e., the singleantenna relay node was energy constrained and had to harvest energy from the single-antenna source firstly, and then exploited the harvested energy to forward the source information to the single-antenna destination under the case of no direct link. Under this scene, [9] firstly proposed the time switching-based relaying (TSR) protocol and the power splitting-based relaying (PSR) protocol. For the TSR protocol, energy harvesting and information 
reception at the relay have taken different time slots . For the PSR protocol, the received signal was split into two streams, one stream got into the energy harvesting circuit and the other got into the information receiver. Subsequently, [10] creatively proposed the hybrid relaying (HR) protocol by combining the advantages of the TSR and PSR protocols. By joint optimizing the TS and PS ratios, the HR protocol showed its great gain compared with the TSR and PSR protocols. At the same time, [11] analyzed the outage probability and the ergodic capacity under the HR protocol. Assuming existing direct source to destination link, [12] analyzed the outage probability and optimized the network performance under the PSR protocol and showed that the large diversity gain can be obtained by exploiting the direct link. By adjusting the number of subframes for each link, $[13,14]$ set the different information transmission rate at the source and the relay, based on which $[13,14]$ further proposed several generalized HR protocols.

Based on the classic three-node model, researchers put forward kinds of novel ideas, techniques, and scenarios for WPCN. Specifically, [15] proposed the idea of exploiting full-duplex (FD) technique to implement self-energy recycling at the multi-antenna relay, so that an additional energy can be obtained by the relay. Luo et al. and Chen et al. $[16,17]$ proposed the idea of allowing the source harvesting energy from the relay in the relaying phase, so that the energy efficiency can be improved greatly. $\mathrm{Li}$ and Rong $[18,19]$ proposed the idea of setting different transmit power in the WET phase and wireless information transmission (WIT) phase, then analyzed a generalized TSR protocol and showed its great gain compared with the traditional TSR protocol. In [20], the idea of channel condition-based adaptive PS at each antenna of the relay was proposed, and the problem of joint PS ratio and power allocation at each transmit antenna of the relay to maximize the throughput was solved. In [21], the HR protocol was applied in the multi-hop relays network. In [22], the effect of co-channel interference (CCI) at the relay node on the ergodic and outage capacities was investigated. Interestingly, [22] found that CCI may be favorable, as CCI also contributed the larger harvested energy except for causing inconvenience for information decoding at the destination. Liu and Wang [23] considered the orthogonal frequency-division multiplexing (OFDM)based WPCN, in which all subcarriers were split into two parts at the relay, one for energy harvesting and one for information reception. [24] considered the multipleinput-multi-output (MIMO) FD-based WPCN, then analyzed and optimized the throughput. [25] investigated the nonorthogonal multiple access (NOMA)-based WPCN, in which the user near the single-antenna source first decoded its needed information from the received signal, then exploited the harvested energy to help forward the information of the far user. Xu et al. [26] aimed to optimize the joint design of beamforming at the multi-antenna source and the PS ratio at the near user in the NOMA based WPCN for the purpose of maximizing the throughput of the near user while satisfying the Quality of Service (QoS) of the far user. Lee et al., Dong et al., Niu et al., and Mei et al. [27-30] aimed to improve the security of WPCN by exploiting beamforming, cooperative jamming, or artificial noise technique.

In view of the prior works, most assumed that there was only one relay assisting the transmission. This setting was easy and would cause large performance loss, as the channel capacity of the second hop (from the relay to the destination) suffered from twice channel fading compared with the case that the relay was energy rich. By placing multiple relays between the source and the destination and exploiting all relays for information forwarding, the channel capacity of the second hop can be enhanced significantly. Meanwhile, there would be no necessity to consume more power, as the harvested energy of all relays will still come from the same RF signal. In addition, multiple antenna technique, as a powerful tool for improving the energy transfer efficiency and the spatial diversity gain, is expected to play an important role in WPCN. Therefore, under the condition that the source is equipped with multiple antennas and there are multiple relays, to maximize the throughput, the joint design of beamforming and time switching/power splitting needs careful consideration and is not a trivial work, which is the motivation for writing this paper.

In this paper, we consider a dual-relay network consisting of one multi-antenna source, two single-antenna relays, and one single-antenna destination. The two energy-constrained relays firstly harvest energy from the RF signal transmitted by the source, and then use this energy to forward the single-stream source information to the destination. Essentially, the main innovations can be summarized as more general system model (with multiple relays [31-33] and a multiantenna source) and more flexible protocol for both WPT and relaying, as compared with the mentioned existing works. Specifically, the main contributions of this work are:

- In our work, we introduce three relaying protocols, i.e., TSR, PSR, and HR protocols, for energy transfer and information transmission in the considered dual-relay system. Although these three protocols have been reported in prior work [10], we should note that the mentioned paper only considered single-relay case. When there exist dual relays and each relay adopt distributed space-time block code for information forwarding, it would become more complex to derive the optimal time switching and 


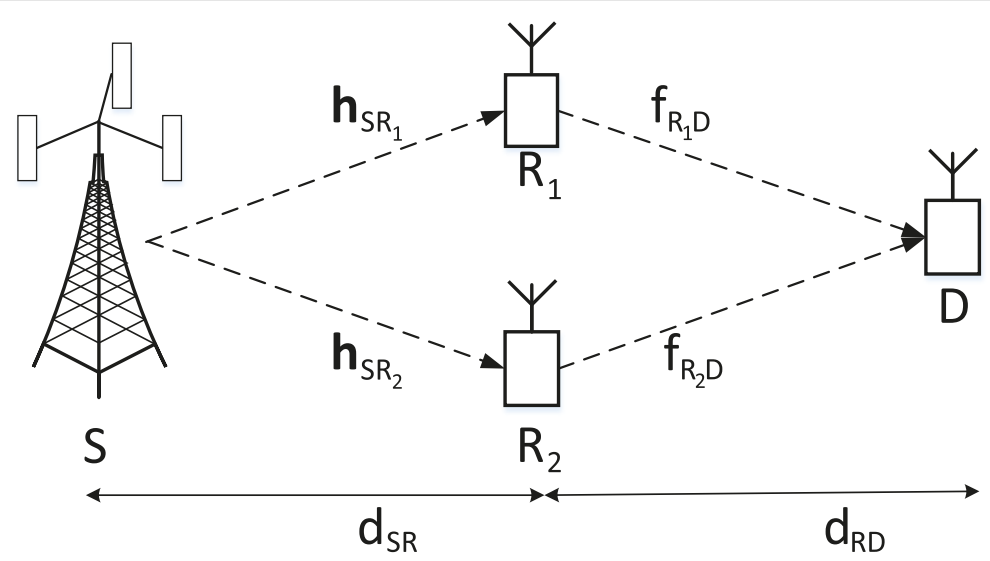

Fig. 1 Considered two-hop dual-relay network model

power splitting ratios. In our work, after complex derivation, we obtain the optimal closed-form solutions.

- We extend the case of single-antenna source to the case of multi-antenna source. Therefore, we have to accordingly design the energy and information beamforming vectors. After some discussion, we find the favorable structure of the optimal energy and information beamforming vectors, which enable us to just optimize single variable for obtaining the optimal solutions instead of resorting to the convex optimization frame such as semi-definite programming (SDP) [34-38]. However, the optimization process for the single variable is not trivial and requires numerical analysis. In our work, we provide the detailed analysis process for finding the optimal energy and information beamformers.

- We provide numerical insights on each protocol and further give the detailed analysis for the effect of the time switching ratio, the power splitting ratio, and the relays' locations on the achievable throughput performance, which are omitted in prior works.

Notation: $(\cdot)^{*},(\cdot)^{T}$ and $(\cdot)^{H}$ denote the complex conjugate, matrix transpose and matrix conjugate transpose, respectively. $\left|h_{X Y}\right|$ denotes the absolute value of a scalar $h_{X Y} \cdot\left\|\mathbf{h}_{X Y}\right\|$ denotes the Frobenius norm of a complex vector $\mathbf{h}_{X Y}$. $\mathbf{I}$ is the identity matrix, $\Pi_{\mathbf{X}}=\mathbf{X}\left(\mathbf{X}^{H} \mathbf{X}\right)^{-1} \mathbf{X}^{H}$ denotes the orthogonal projection onto the column space of $\mathbf{X}$, and $\Pi_{\mathbf{X}}^{\perp}=\mathbf{I}-\Pi_{\mathbf{X}}$ denotes the orthogonal projection onto the orthogonal complement of column space $\mathbf{X}$. $\mathcal{C N}(0,1)$ denotes a scalar complex Gaussian distribution with zero mean and unit variance.

\section{Methods}

The rest of this paper is organized as follows: Section 3 describes the system model. Section 4 gives the optimization process for the TSR protocol. Section 5 gives the optimization process for the PSR protocol. Section 6 gives the optimization process for the HR protocol. Section 7 presents the simulation results and provides some insightful details. Finally, conclusion is given in Section 8.

\section{System models}

As shown in Fig. 1, we consider a wireless-powered dualrelay network, where a source $(S)$ equipped with $N(N \geq$ 2) antennas aims to transmit information to the singleantenna destination $(D)$ with the assistance of two singleantenna relays $R_{1}$ and $R_{2}{ }^{1}$ All nodes are operated in half-duplex (HD) mode. Denote $N \times 1$ vector $\mathbf{h}_{S R_{i}}(i \in$ $\{1,2\}$ ) as the $S$ to $R_{i}$ channel coefficients with entries being independent and identically distributed (i.i.d.) $\mathcal{C N}(0,1)$ random variables. Similarly, denote $f_{R_{i} D} \sim \mathcal{C N}(0,1)$ as the channel coefficient from $R_{i}$ to $D$. The distances from $S$ to $R_{i}$ and from $R_{i}$ to $D$ are denoted by $d_{S R_{i}}$ and $d_{R_{i} D}$, respectively. We consider a linear topology, i.e., $R_{1}$ and $R_{2}$ are located on a straight line between $S$ and $D$; thus, we have $d_{S R_{1}}=d_{S R_{2}}=d_{S R}$ and $d_{R_{1} D}=d_{R_{2} D}=$ $d_{S D}-d_{S R}$. Throughout this paper, we make the following assumptions:

- There is no direct link between $S$ and $D$, which may be resulted from the deep shadowing or shielding effect caused by the obstacles [41].

- It is assumed that all channel gains keep constant during each transmission block (TB) but change independently from one TB to another.

- The relay nodes $R_{1}$ and $R_{2}$ have no external power supply and would harvest energy from the source RF

\footnotetext{
${ }^{1}$ In our work, we considered the dual-relay case because of the following reasons: the dual-relay network, i.e., diamond network, has been discussed in numerical classical works, such as [38-40], which all demonstrated that the dual-relay network was worth studying and also provided valuable guidance for us. In addition, to the best knowledge of the authors, there are no related works that have employed the energy harvesting technique in the dual-relay network, where the source is equipped with multiple antennas. This motivates us to carry out detailed research for the energy harvesting based dual-relay network.
} 


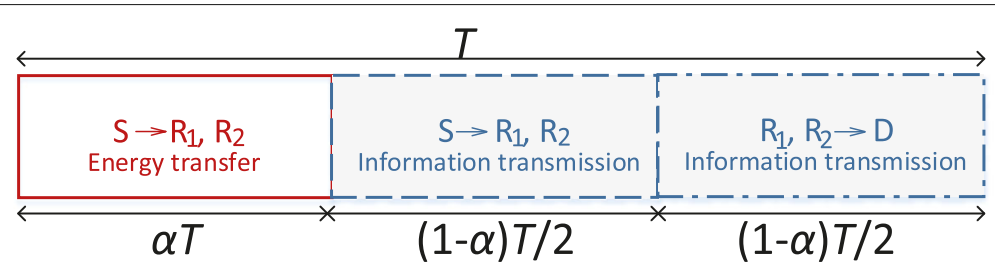

Fig. 2 Diagram of the TSR protocol

signal firstly, then exhaust the harvested energy to help the source information transmission based on decode-and-forward (DF) technique.

- As the necessary conditions for designing optimal scheduling strategies (such as the design of energy and information beamforming vectors at $S$, the TS, and $P S$ ratios) under different relaying protocols, the global channel state information (CSI) are assumed to be available at all nodes, which can be realized through various methods, e.g., the pilot-assisted reverse-link channel training [42].

Based on the network model and above assumptions, we introduce three different relaying protocols, i.e., TSR, PSR, and HR to drive the energy harvesting at the relays and information transmission from the source to the destination for each TB. More importantly, in order to maximize the network throughput, we present a detailed investigation on the optimal design of beamformers at the source, the TS ratio, the PS ratio, and the joint TS\&PS ratio under these protocols in the following sections.

\section{Time switching-based relaying (TSR) protocol}

As shown in Fig. 2, in the TSR protocol, each TB with time length $T$ is divided into three phases: the first phase with time length $\alpha T(\alpha \in[0,1])$ is allocated to the energy transfer from $S$ to $R_{1}$ and $R_{2}$. The second phase with time length $(1-\alpha) T / 2$ is allocated to the information transmission from $S$ to $R_{1}$ and $R_{2}$. The third phase with time length $(1-\alpha) T / 2$ is allocated to the information transmission from $R_{1}$ and $R_{2}$ to $D$. For simplification, $T$ is set to 1 in this paper.

It is worthy noted that in the relays' transmission phase, the two relays would forward the source information to $D$ with the help of distributed space-time block code (DSTC) [43].

Denote the transmit power of $S$ during the first and second phase of each TB as $P_{S}$. We assume that the energy harvested by $R_{1}$ and $R_{2}$ from the noise is negligible. Therefore, the harvested energy by $R_{i}$ after the first phase can be written as

$$
E_{R_{i}}=\frac{\eta \alpha P_{S}\left|\mathbf{h}_{S R_{i}}^{T} \mathbf{w}_{1}\right|^{2}}{d_{S R}^{m}}
$$

where $i \in\{1,2\}, 0<\eta<1$ denotes the energy conversion efficiency, $m$ denotes the path loss factor, and $\mathbf{w}_{1}$ is the $N \times 1$ energy beamforming vector employed at $S$.

In the second phase, $S$ employs the $N \times 1$ beamforming vector $\mathbf{w}_{2}{ }^{2}$ for information transmission. Hence, the received SNR at $R_{i}$ after the second phase is given by

$$
\gamma_{S R_{i}}=\frac{P_{S}\left|\mathbf{h}_{S R_{i}}^{T} \mathbf{w}_{2}\right|^{2}}{d_{S R}^{m} N_{0}}
$$

where $N_{0}$ denotes the noise power.

Note that when the DF technique is adopted at $R_{1}$ and $R_{2}$, the SNR of the first hop (from the source to the relays) is given $\mathrm{by}^{3}$

$$
\begin{aligned}
\gamma_{D F, 1} & =\min \left(\gamma_{S R_{1}}, \gamma_{S R_{2}}\right) \\
& =\min \left(A_{1}\left|\mathbf{h}_{S R_{1}}^{T} \mathbf{w}_{2}\right|^{2}, A_{1}\left|\mathbf{h}_{S R_{2}}^{T} \mathbf{w}_{2}\right|^{2}\right)
\end{aligned}
$$

where $A_{1}=\frac{P_{S}}{d_{S R}^{m} N_{0}}$.

In the third phase, $R_{1}$ and $R_{2}$ exhaust their harvested energy to forward the source information to $D$. The transmit power of $R_{i}$ is given by $P_{R_{i}}=\frac{E_{R_{i}}}{(1-\alpha) / 2}=\frac{2 \eta \alpha P_{S}\left|\mathbf{h}_{S R_{i}}^{T} \mathbf{w}_{1}\right|^{2}}{(1-\alpha) d_{S R}^{m}}$. Hence, the SNR of the second hop (from the relays to the destination) can be written as

$$
\begin{aligned}
\gamma_{D F, 2}= & \frac{P_{R_{1}}\left|f_{R_{1} D}\right|^{2}}{d_{R D}^{m} N_{0}}+\frac{P_{R_{2}}\left|f_{R_{2} D}\right|^{2}}{d_{R D}^{m} N_{0}} \\
= & \frac{2 \eta \alpha P_{S}\left|\mathbf{h}_{S R_{1}}^{T} \mathbf{w}_{1}\right|^{2}\left|f_{R_{1} D}\right|^{2}}{(1-\alpha) d_{S R}^{m} d_{R D}^{m} N_{0}} \\
& +\frac{2 \eta \alpha P_{S}\left|\mathbf{h}_{S R_{2}}^{T} \mathbf{w}_{1}\right|^{2}\left|f_{R_{2} D}\right|^{2}}{(1-\alpha) d_{S R}^{m} d_{R D}^{m} N_{0}} \\
= & \frac{\alpha}{1-\alpha}\left(A_{2}\left|\mathbf{h}_{S R_{1}}^{T} \mathbf{w}_{1}\right|^{2}+A_{3}\left|\mathbf{h}_{S R_{2}}^{T} \mathbf{w}_{1}\right|^{2}\right)
\end{aligned}
$$

where $A_{2}=\frac{2 \eta P_{S}\left|f_{R_{1} D}\right|^{2}}{d_{S R}^{m} d_{R D}^{m} N_{0}}$ and $A_{3}=\frac{2 \eta P_{S}\left|f_{R_{2} D}\right|^{2}}{d_{S R}^{m} d_{R D}^{m} N_{0}}$.

\footnotetext{
${ }^{2}$ To keep consistency, in the following sections, we also use $\mathbf{w}_{1}$ and $\mathbf{w}_{2}$ to represent the energy beamforming vector and information beamforming vector, respectively.

${ }^{3}$ Because that the two relays all participant in the transmission process, the efficient SNR of the first hop must be determined by the minimal received SNR by the two relays.
} 
Therefore, the achievable end-to-end throughput is given by

$$
R_{D F}=\frac{1-\alpha}{2} \log _{2}\left(1+\min \left(\gamma_{D F, 1}, \gamma_{D F, 2}\right)\right)
$$

Our objective is to maximize $R_{D F}$ by optimizing $\alpha, \mathbf{w}_{1}$, and $\mathbf{w}_{2}$. The optimization problem can be formulated as

$$
\begin{aligned}
\text { P1 : } & \max _{\alpha, \mathbf{w}_{1}, \mathbf{w}_{2}} R_{D F} \\
& \text { s.t. } \quad \alpha \in[0,1],\left\|\mathbf{w}_{1}\right\|^{2}=\left\|\mathbf{w}_{2}\right\|^{2}=1 .
\end{aligned}
$$

To proceed, we first have the following Proposition.

Proposition 1 The optimal beamforming vector $\mathbf{w}_{i}(i \in$ $\{1,2\})$ for the problem $\mathbf{P 1}$ has the following structure

$$
\mathbf{w}_{i}^{o p t}=x_{i} \frac{\prod_{\mathbf{h}_{S R_{1}}^{*}} \mathbf{h}_{S R_{2}}^{*}}{\left\|\prod_{\mathbf{h}_{S R_{1}}} \mathbf{h}_{S R_{2}}\right\|}+\sqrt{1-x_{i}^{2}} \frac{\prod_{\mathbf{h}_{S R_{1}}^{*}}^{\perp} \mathbf{h}_{S R_{2}}^{*}}{\left\|\prod_{\mathbf{h}_{S R_{1}}}^{\perp} \mathbf{h}_{S R_{2}}\right\|}
$$

where $x_{i}$ is a real number in $[0,1]$.

Proof Same with the objective function shown in Eq. (2) of [44], $R_{D F}$ is nondecreasing with respect to $\left|\mathbf{h}_{S R_{1}}^{T} \mathbf{w}_{1}\right|^{2}$, $\left|\mathbf{h}_{S R_{2}}^{T} \mathbf{w}_{1}\right|^{2},\left|\mathbf{h}_{S R_{1}}^{T} \mathbf{w}_{2}\right|^{2}$, and $\left|\mathbf{h}_{S R_{2}}^{T} \mathbf{w}_{2}\right|^{2}$. And the beamforming vectors $\mathbf{w}_{i}$ in fact should play the role for balancing the values of $\left|\mathbf{h}_{S R_{1}}^{T} \mathbf{w}_{i}\right|^{2}$ and $\left|\mathbf{h}_{S R_{2}}^{T} \mathbf{w}_{i}\right|^{2}$ as far as possible. Therefore, the process for proving Proposition 1 can be obtained by resorting to the proof of Lemma 1 presented in [44].

By substituting (7) into (6), the problem $\mathbf{P 1}$ is rewritten as

$$
\begin{aligned}
& \text { P1: } \max _{\alpha, x_{1}, x_{2}} \frac{1-\alpha}{2} \log _{2}\left(1+\min \left(f_{1}\left(x_{1}\right), \frac{\alpha}{1-\alpha} f_{2}\left(x_{2}\right)\right)\right) \\
& \text { s.t. } \alpha \in[0,1], 0 \leq x_{1}, x_{2} \leq 1 .
\end{aligned}
$$

where $f_{1}\left(x_{1}\right)=\min \left(A_{1} a^{2} x_{1}^{2}, A_{1}\left(b x_{1}+c \sqrt{1-x_{1}^{2}}\right)^{2}\right)$, $f_{2}\left(x_{2}\right)=A_{2} a^{2} x_{2}^{2}+A_{3}\left(b x_{2}+c \sqrt{1-x_{2}^{2}}\right)^{2}$, $a=\frac{\left|\mathbf{h}_{S R_{1}}^{T} \prod_{\mathbf{h}_{S R_{1}}^{*}} \mathbf{h}_{S R_{2}}^{*}\right|}{\left\|\prod_{\mathbf{h}_{S R_{1}}} \mathbf{h}_{S R_{2}}\right\|}, \quad b=\left\|\prod_{\mathbf{h}_{S R_{1}}} \mathbf{h}_{S R_{2}}\right\|$ and $c=\left\|\prod_{\mathbf{h}_{S R_{1}}}^{\perp} \mathbf{h}_{S R_{2}}\right\|$.

As we can see, when $\alpha$ is fixed, maximizing $R_{D F}$ is equivalent to maximizing $\min \left(f_{1}\left(x_{1}\right), \frac{\alpha}{1-\alpha} f_{2}\left(x_{2}\right)\right)$. Observing that $f_{1}\left(x_{1}\right)$ is independent of $f_{2}\left(x_{2}\right)$, hence next we aim to find the optimal $x_{1}$ and $x_{2}$ to maximize $f_{1}\left(x_{1}\right)$ and $f_{2}\left(x_{2}\right)$, respectively.
- The process of finding $x_{1}^{*}$ : based on the conclusion shown in the Proposition 2 of [45], the optimal $x_{1}^{*}$ is given as follows:

$$
x_{1}^{*}=\left\{\begin{array}{cc}
1 & a<b \\
\frac{c}{\sqrt{c^{2}+(a-b)^{2}}} & b \leq a<\frac{b^{2}+c^{2}}{b} \\
\frac{b}{\sqrt{b^{2}+c^{2}}} & a \geq \frac{b^{2}+c^{2}}{b}
\end{array}\right.
$$

- The process of finding $x_{2}^{*}: f_{2}\left(x_{2}\right)$ can be further written as $f_{2}\left(x_{2}\right)=$ $\left(A_{2} a^{2}+A_{3} b^{2}-A_{3} c^{2}\right) x_{2}^{2}+2 A_{3} b c x_{2} \sqrt{1-x_{2}^{2}}+A_{3} c^{2}$. When $A_{2} a^{2}+A_{3} b^{2}-A_{3} c^{2} \geq 0$, by letting the first derivative of $f_{2}\left(x_{2}\right)$ equaling 0 , we have the unique $\operatorname{root} \widehat{x}_{2}=\sqrt{\frac{1+\sqrt{\frac{u^{2}}{u^{2}+4 v^{2}}}}{2}}$, where $u=2\left(A_{2} a^{2}+A_{3} b^{2}-A_{3} c^{2}\right)$ and $v=2 A_{3} b c$. Moreover, because that $f_{2}{ }^{\prime}\left(x_{2}\right)>0$ and $f_{2}{ }^{\prime}\left(x_{2}\right)<0$ when $x \in\left[0, \widehat{x}_{2}\right)$ and $x \in\left(\widehat{x}_{2}, 1\right]$, we can claim that $f_{2}\left(x_{2}\right)$ achieves its maximum at the point $\widehat{x}_{2}$. When $A_{2} a^{2}+A_{3} b^{2}-A_{3} c^{2}<0$, due to the fact that $\left(A_{2} a^{2}+A_{3} b^{2}-A_{3} c^{2}\right) x_{2}^{2}$ and $2 A_{3} b c x_{2} \sqrt{1-x_{2}^{2}}$ are two concave functions, $f_{2}\left(x_{2}\right)$ must be a concave function and achieves its maximum value at the point $\widehat{x}_{2}=\sqrt{\frac{1-\sqrt{\frac{u^{2}}{u^{2}+4 v^{2}}}}{2}}$. Therefore, the optimal $x_{2}$ is given as follows:

$$
x_{2}^{*}=\left\{\begin{array}{l}
\sqrt{\frac{1+\sqrt{\frac{u^{2}}{u^{2}+4 v^{2}}}}{2}} A_{2} a^{2}+A_{3} b^{2}-A_{3} c^{2} \geq 0 \\
\sqrt{\frac{1-\sqrt{\frac{u^{2}}{u^{2}+4 v^{2}}}}{2}} A_{2} a^{2}+A_{3} b^{2}-A_{3} c^{2}<0
\end{array}\right.
$$

Based on $f_{1}\left(x_{1}^{*}\right)$ and $f_{2}\left(x_{2}^{*}\right)$, the problem $\mathbf{P} \mathbf{1}$ is rewritten as

$$
\begin{aligned}
& \mathbf{P 1}: \max _{\alpha} \frac{1-\alpha}{2} \log _{2}\left(1+\min \left(f_{1}\left(x_{1}^{*}\right), \frac{\alpha}{1-\alpha} f_{2}\left(x_{2}^{*}\right)\right)\right) \\
& \text { s.t. } \quad \alpha \in[0,1] .
\end{aligned}
$$

To find the optimal $\alpha$, we first let $f_{1}\left(x_{1}^{*}\right)=\frac{\alpha}{1-\alpha} f_{2}\left(x_{2}^{*}\right)$ and obtain the root of which equaling $\bar{\alpha}=\frac{f_{1}\left(x_{1}^{*}\right)}{f_{1}\left(x_{1}^{*}\right)+f_{2}\left(x_{2}^{*}\right)}$. As we can see, with the value of $\alpha$ increasing in the interval $[\bar{\alpha}, 1], \min \left(f_{1}\left(x_{1}^{*}\right), \frac{\alpha}{1-\alpha} f_{2}\left(x_{2}^{*}\right)\right)$ would equal $f_{1}\left(x_{1}^{*}\right)$ (the obtained maximal SNR of the first hop) and $R_{D F}$ would constantly decrease. Hence, the optimal solution must be smaller than or equal to $\bar{\alpha}$. Observing that $\frac{1-\alpha}{2} \log _{2}\left(1+\frac{\alpha}{1-\alpha} f_{2}\left(x_{2}^{*}\right)\right)$ is a concave function with respect to $\alpha$ and the maximal value of this function is 
obtained at $\widehat{\alpha}=\frac{e^{\mathbb{W}\left(\frac{f_{2}\left(x_{2}^{*}\right)-1}{e}\right)+1}-1}{f_{2}\left(x_{2}^{*}\right)+e^{\mathbb{W}\left(\frac{f_{2}\left(x_{2}^{*}\right)-1}{e}\right)+1}-1}[46]$, where $\mathbb{W}(\cdot)$ is the Lambert function [47]. Hence, the optimal $\alpha$ for $\mathbf{P 1}$ is given by

$$
\begin{aligned}
\alpha^{*} & =\min (\widehat{\alpha}, \bar{\alpha}) \\
& =\min \left(\frac{e^{\mathbb{W}\left(\frac{f_{2}\left(x_{2}^{*}\right)-1}{e}\right)+1}-1}{f_{2}\left(x_{2}^{*}\right)+e^{\mathbb{W}\left(\frac{f_{2}\left(x_{2}^{*}\right)-1}{e}\right)+1}-1}, \frac{f_{1}\left(x_{1}^{*}\right)}{f_{1}\left(x_{1}^{*}\right)+f_{2}\left(x_{2}^{*}\right)}\right)
\end{aligned}
$$

Remark 1 With $\alpha$ increasing in the interval $[0, \bar{\alpha}]$, the efficient information transmission time would decrease linearly and the SNR of the second hop would increase at the same time. This tradeoff makes the optimal $\alpha$ exist. However, $\gamma_{D F, 2}$ always does not exceed $\gamma_{D F, 1}$ when the throughput is maximized. In the HR protocol as introduced in Section 6, we can see that in the second phase, each relay could adaptively split some fraction of the received signal as an additional harvested energy (expected the harvested energy in the first phase) to further improve the SNR of the second hop and reduce the SNR of the first hop. By selecting suitable splitting ratio, $\gamma_{D F, 1}$ and $\gamma_{D F, 2}$ would be balanced as far as possible and the efficient SNR would be improved greatly. Hence, without decreasing the information transmission time, the HR protocol offsets the shortcoming of the $T S R$ protocol efficiently and achieves the superior throughput performance, as shown in the simulations.

\section{Power splitting-based relaying (PSR) protocol}

As shown in Fig. 3, in the PSR protocol, each TB with time length $T$ is divided into two phases: the first phase with time length $T / 2$ is allocated to the information transmission from $S$ to $R_{1}$ and $R_{2}$. A fraction $\left(\lambda_{i} \in[0,1], i \in\{1,2\}\right)$ of the received signals is used for energy harvesting at $R_{i}$, and the fraction $\left(1-\lambda_{i}\right)$ of the received signal is used for information reception at $R_{i}$. The second phase with time length $T / 2$ is allocated to the information transmission from $R_{1}$ and $R_{2}$ to $D$ concurrently.

Hence, the received SNR at $R_{i}$ after the first phase is given by

$$
\gamma_{S R_{i}}=\frac{P_{S}\left|\mathbf{h}_{S R_{i}}^{T} \mathbf{w}_{2}\right|^{2}\left(1-\lambda_{i}\right)}{d_{S R}^{m} N_{0}}
$$

and the SNR of the first hop is given by

$$
\begin{aligned}
\gamma_{D F, 1} & =\min \left(\gamma_{S R_{1}}, \gamma_{S R_{2}}\right) \\
& =\min \left(A_{1}\left|\mathbf{h}_{S R_{1}}^{T} \mathbf{w}_{2}\right|^{2}\left(1-\lambda_{1}\right), A_{1}\left|\mathbf{h}_{S R_{2}}^{T} \mathbf{w}_{2}\right|^{2}\left(1-\lambda_{2}\right)\right)
\end{aligned}
$$

Observing that the transmit power of $R_{i}$ is $P_{R_{i}}=\frac{E_{R_{i}}}{1 / 2}=$ $\frac{\eta P_{S}\left|h_{S R_{i}}^{T} \mathbf{w}_{2}\right|^{2} \lambda_{i}}{d_{S R}^{m}}$. Hence, the SNR of the second hop can be written as

$$
\begin{aligned}
\gamma_{D F, 2}= & \frac{P_{R_{1}}\left|f_{R_{1} D}\right|^{2}}{d_{R D}^{m} N_{0}}+\frac{P_{R_{2}}\left|f_{R_{2} D}\right|^{2}}{d_{R D}^{m} N_{0}} \\
= & \frac{\eta P_{S}\left|\mathbf{h}_{S R_{1}}^{T} \mathbf{w}_{2}\right|^{2} \lambda_{1}\left|f_{R_{1} D}\right|^{2}}{d_{S R}^{m} d_{R D}^{m} N_{0}} \\
& +\frac{\eta P_{S}\left|\mathbf{h}_{S R_{2}}^{T} \mathbf{w}_{2}\right|^{2} \lambda_{2}\left|f_{R_{2} D}\right|^{2}}{d_{S R}^{m} d_{R D}^{m} N_{0}} \\
= & B_{1}\left|\mathbf{h}_{S R_{1}}^{T} \mathbf{w}_{2}\right|^{2} \lambda_{1}+B_{2}\left|\mathbf{h}_{S R_{2}}^{T} \mathbf{w}_{2}\right|^{2} \lambda_{2}
\end{aligned}
$$

where $B_{1}=\frac{\eta P_{S}\left|f_{R_{1} D}\right|^{2}}{d_{S R}^{m} d_{R D}^{m} N_{0}}$ and $B_{2}=\frac{\eta P_{S}\left|f_{R_{2} D}\right|^{2}}{d_{S R}^{m} d_{R D}^{m} N_{0}}$.

Therefore, the achievable end-to-end throughput is given by

$$
R_{D F}=\frac{1}{2} \log _{2}\left(1+\min \left(\gamma_{D F, 1}, \gamma_{D F, 2}\right)\right)
$$

Next, we aim to maximize $R_{D F}$ by optimizing $\lambda_{1}, \lambda_{2}$ and $\mathbf{w}_{2}$. Then, the optimization problem can be formulated as

$$
\begin{aligned}
\text { P2: } & \max _{\lambda_{1}, \lambda_{2}, \mathbf{w}_{2}} R_{D F} \\
& \text { s.t. } \quad \lambda_{1}, \lambda_{2} \in[0,1],\left\|\mathbf{w}_{2}\right\|^{2}=1 .
\end{aligned}
$$

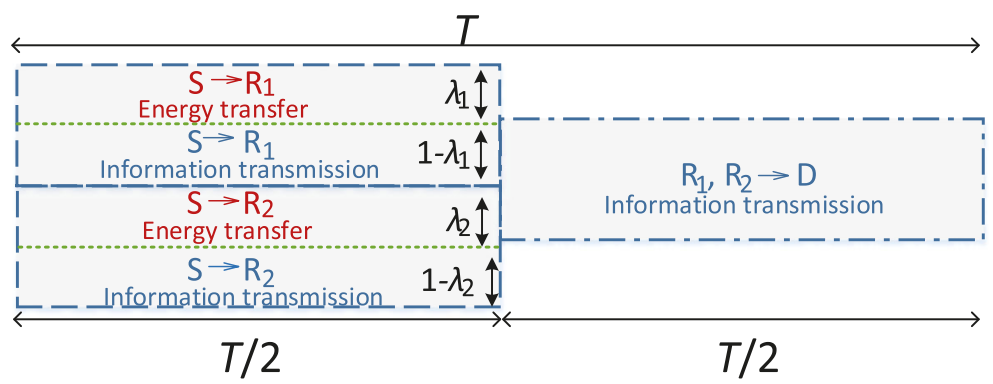

Fig. 3 Diagram of the PSR protocol 
For the PSR protocol, the optimal solution must happen at $\gamma_{D F, 1}=\gamma_{D F, 2}[10]$, which indicates that the following equation is established:

$$
\begin{gathered}
\min \left(A_{1}\left|\mathbf{h}_{S R_{1}}^{T} \mathbf{w}_{2}\right|^{2}\left(1-\lambda_{1}\right), A_{1}\left|\mathbf{h}_{S R_{2}}^{T} \mathbf{w}_{2}\right|^{2}\left(1-\lambda_{2}\right)\right) \\
=B_{1}\left|\mathbf{h}_{S R_{1}}^{T} \mathbf{w}_{2}\right|^{2} \lambda_{1}+B_{2}\left|\mathbf{h}_{S R_{1}}^{T} \mathbf{w}_{2}\right|^{2} \lambda_{2}
\end{gathered}
$$

Moreover, one can prove that the received SNR at $R_{1}$ and $R_{2}$ should be same. Hence, the following condition should be satisfied at the optimum:

$$
\begin{aligned}
\gamma_{\text {ete }} & =A_{1}\left|\mathbf{h}_{S R_{1}}^{T} \mathbf{w}_{2}\right|^{2}\left(1-\lambda_{1}\right)=A_{1}\left|\mathbf{h}_{S R_{2}}^{T} \mathbf{w}_{2}\right|^{2}\left(1-\lambda_{2}\right) \\
& =B_{1}\left|\mathbf{h}_{S R_{1}}^{T} \mathbf{w}_{2}\right|^{2} \lambda_{1}+B_{2}\left|\mathbf{h}_{S R_{2}}^{T} \mathbf{w}_{2}\right|^{2} \lambda_{2}
\end{aligned}
$$

where $\gamma_{\text {ete }}$ is the end-to-end SNR. Based on (19), we have

$$
\begin{aligned}
\gamma_{\text {ete }}= & B_{1}\left|\mathbf{h}_{S R_{1}}^{T} \mathbf{w}_{2}\right|^{2}\left(1-\frac{\gamma_{\text {ete }}}{A_{1}\left|\mathbf{h}_{S R_{1}}^{T} \mathbf{w}_{2}\right|^{2}}\right) \\
& +B_{2}\left|\mathbf{h}_{S R_{2}}^{T} \mathbf{w}_{2}\right|^{2}\left(1-\frac{\gamma_{\text {ete }}}{A_{1}\left|\mathbf{h}_{S R_{2}}^{T} \mathbf{w}_{2}\right|^{2}}\right) \\
= & B_{1}\left|\mathbf{h}_{S R_{1}}^{T} \mathbf{w}_{2}\right|^{2}+B_{2}\left|\mathbf{h}_{S R_{2}}^{T} \mathbf{w}_{2}\right|^{2}-\left(\frac{B_{1}}{A_{1}}+\frac{B_{2}}{A_{1}}\right) \gamma_{\text {ete }}
\end{aligned}
$$

therefore, $\gamma_{\text {ete }}$ is given by

$$
\begin{aligned}
\gamma_{\text {ete }} & =\frac{B_{1}\left|\mathbf{h}_{S R_{1}}^{T} \mathbf{w}_{2}\right|^{2}+B_{2}\left|\mathbf{h}_{S R_{2}}^{T} \mathbf{w}_{2}\right|^{2}}{1+\frac{B_{1}}{A_{1}}+\frac{B_{2}}{A_{1}}} \\
& =B_{3}\left|\mathbf{h}_{S R_{1}}^{T} \mathbf{w}_{2}\right|^{2}+B_{4}\left|\mathbf{h}_{S R_{2}}^{T} \mathbf{w}_{2}\right|^{2}
\end{aligned}
$$

where $B_{3}=\frac{B_{1}}{1+\frac{B_{1}}{A_{1}}+\frac{B_{2}}{A_{1}}}$ and $B_{4}=\frac{B_{2}}{1+\frac{B_{1}}{A_{1}}+\frac{B_{2}}{A_{1}}}$.

Combining the constraint in (17), the efficient SNR is given by

$$
\begin{aligned}
\gamma_{\text {eff }}=\min & \left(B_{3}\left|\mathbf{h}_{S R_{1}}^{T} \mathbf{w}_{2}\right|^{2}+B_{4}\left|\mathbf{h}_{S R_{2}}^{T} \mathbf{w}_{2}\right|^{2},\right. \\
& \left.\min \left(A_{1}\left|\mathbf{h}_{S R_{1}}^{T} \mathbf{w}_{2}\right|^{2}, A_{1}\left|\mathbf{h}_{S R_{2}}^{T} \mathbf{w}_{2}\right|^{2}\right)\right)
\end{aligned}
$$

Hence, maximizing $R_{D F}$ is equivalent to maximizing $\gamma_{\text {eff }}$. Formally, the optimization problem can be formulated as

$$
\begin{aligned}
\text { P3: } & \max _{\mathbf{w}_{2}} \gamma_{\text {eff }} \\
\text { s.t. } & \left\|\mathbf{w}_{2}\right\|^{2}=1 .
\end{aligned}
$$

It is easy to observe that the optimal beamforming vector $\mathbf{w}_{2}^{\text {opt }}$ for maximizing $\gamma_{\text {eff }}$ admits the same form as in (7). Hence, $\mathbf{P 3}$ can be rewritten as

$$
\begin{gathered}
\text { P3: } \max _{x} \min \left(g_{1}(x), g_{2}(x)\right) \\
\text { s.t. } \quad 0 \leq x \leq 1 .
\end{gathered}
$$

where $g_{1}(x)=B_{3} a^{2} x^{2}+B_{4}\left(b x+c \sqrt{1-x^{2}}\right)^{2}$ and $g_{2}(x)=$ $\min \left(A_{1} a^{2} x^{2}, A_{1}\left(b x+c \sqrt{1-x^{2}}\right)^{2}\right)$.

To obtain the optimal $x$ that maximizes $\min \left(g_{1}(x), g_{2}(x)\right)$, next we discuss three cases in term of different solution with respect to $x$ that maximizes $g_{2}(x)$ :

\subsection{Case 1: $a<b$}

Note that $A_{1} a^{2} x^{2}$ would increase and $A_{1}\left(b x+c \sqrt{1-x^{2}}\right)^{2}$ would first increase and then decrease with the increasing $x \in[0,1]$. In this case, there is no cross point between $A_{1} a^{2} x^{2}$ and $A_{1}\left(b x+c \sqrt{1-x^{2}}\right)^{2}$. Hence, $g_{2}(x)=A_{1} a^{2} x^{2}$, as shown by the blue line in Fig. 4a, and the maximum of which is obtained at $\bar{x}=1$.

Same as $f_{2}\left(x_{2}\right), g_{1}(x)$ first increases and then decreases in the interval $[0,1]$ and achieves its maximum at

$$
\widehat{x}=\left\{\begin{array}{l}
\sqrt{\frac{1+\sqrt{\frac{u_{1}^{2}}{u_{1}^{2}+4 v_{1}^{2}}}}{2}} B_{3} a^{2}+B_{4} b^{2}-B_{4} c^{2} \geq 0 \\
\sqrt{\frac{1-\sqrt{\frac{u_{1}^{2}}{u_{1}^{2}+4 v_{1}^{2}}}}{2}} B_{3} a^{2}+B_{4} b^{2}-B_{4} c^{2}<0
\end{array}\right.
$$

where $u_{1}=2\left(B_{3} a^{2}+B_{4} b^{2}-B_{4} c^{2}\right)$ and $v_{1}=2 B_{4} b c$.

Lemma 1 When $a<b$, the optimal solution of $\mathbf{P 3}$ is expressed as

$$
x^{*}=\left\{\begin{array}{cl}
1 & g_{2}(1) \leq g_{1}(1) \\
\sqrt{\frac{2 z_{1} w_{1}+v_{1}^{2}+\sqrt{v_{1}^{4}+4 z_{1} w_{1} v_{1}^{2}-4 v_{1}^{2} w_{1}^{2}}}{2\left(z_{1}^{2}+v_{1}^{2}\right)}} & g_{2}(1)>g_{1}(1), \\
\widehat{\widehat{x}} & g_{2}(\widehat{x}) \leq g_{1}(\widehat{x}) \\
& g_{2}(\widehat{x})>g_{1}(\widehat{x})
\end{array}\right.
$$

where $w_{1}=B_{4} c^{2}$ and $z_{1}=A_{1} a^{2}-B_{3} a^{2}-B_{4}\left(b^{2}-c^{2}\right)$.

Proof As we can see, under this case, there are only three scenarios:

1. In this scenario, $g_{2}(1) \leq g_{1}(1)$. There is no cross point between $g_{1}(x)$ and $g_{2}(x)$, and $g_{1}(x)$ is always greater than $g_{2}(x)$. Hence, $\min \left(g_{1}(x), g_{2}(x)\right)$ achieves its maximal value when $g_{2}(x)$ is maximized, i.e., $x^{*}=1$.

2. In this scenario, $g_{2}(1)>g_{1}(1)$ and $g_{2}(\widehat{x}) \leq g_{1}(\widehat{x})$. There exists one cross point between $g_{1}(x)$ and $g_{2}(x)$, which occurs after the point at which $g_{1}(x)$ achieves 

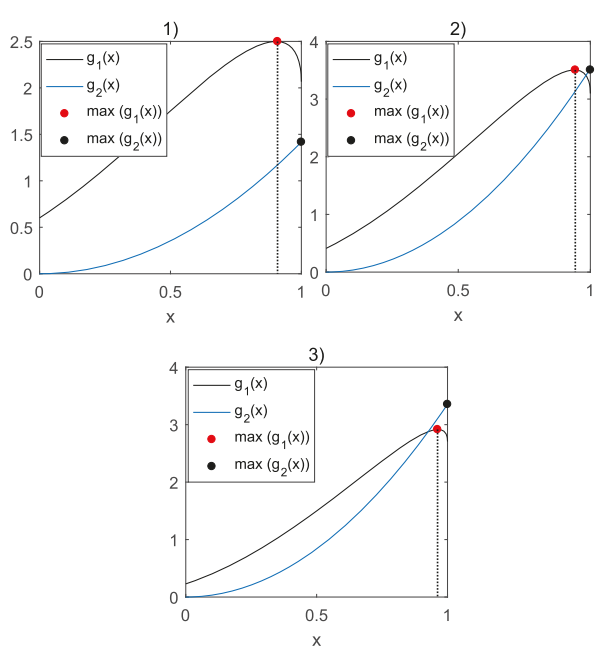

(a)
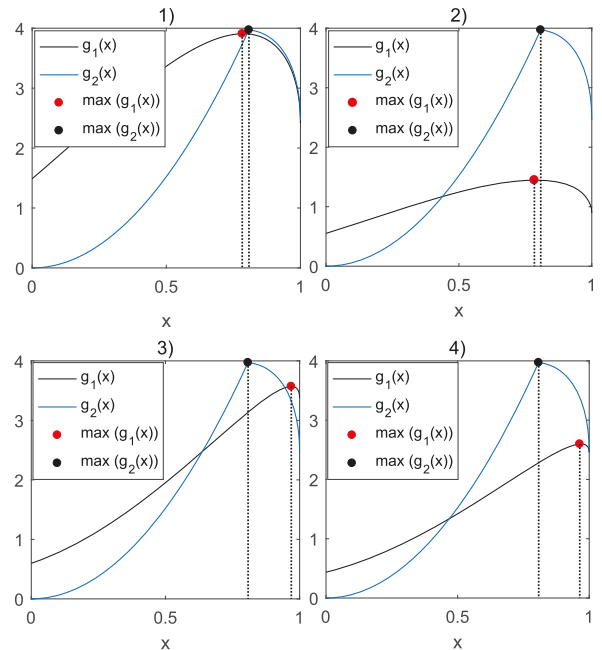

(b)
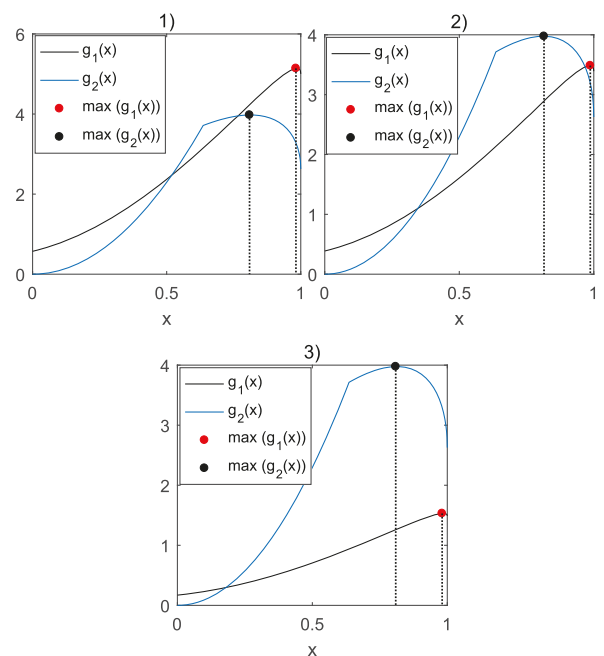

(c)

Fig. 4 a Case 1. b Case 2. c Case 3 its maximal value. Hence, $\min \left(g_{1}(x), g_{2}(x)\right)$ is maximized when $g_{1}(x)=g_{2}(x)$, i.e.,

$x^{*}=\sqrt{\frac{2 z_{1} w_{1}+v_{1}^{2}+\sqrt{v_{1}^{4}+4 z_{1} w_{1} v_{1}^{2}-4 v_{1}^{2} w_{1}^{2}}}{2\left(z_{1}^{2}+v_{1}^{2}\right)}}$.

3. In this scenario, $g_{2}(\widehat{x})>g_{1}(\widehat{x})$. There also exists one cross point between $g_{1}(x)$ and $g_{2}(x)$, which occurs before the point at which $g_{1}(x)$ is maximized. Hence, $\min \left(g_{1}(x), g_{2}(x)\right)$ achieves its maximal value at the point when $g_{1}(x)$ is maximized, i.e., $x^{*}=\widehat{x}$.

5.2 Case 2: $b \leq a<\frac{b^{2}+c^{2}}{b}$

In this case, $g_{2}(x)$ is a piecewise function, as shown by the blue line in Fig. 4b. Obviously, the maximum of $g_{2}(x)$ is obtained at $\bar{x}=\frac{c}{\sqrt{(a-b)^{2}+c^{2}}}$, i.e., the cross point of the functions $A_{1} a^{2} x^{2}$ and $A_{1}\left(b x+c \sqrt{1-x^{2}}\right)^{2}$.

Lemma 2 When $b \leq a<\frac{b^{2}+c^{2}}{b}$, the optimal solution of $P 3$ is expressed as

$$
x^{*}=\left\{\begin{array}{cl}
\sqrt{\frac{2 z_{1} w_{1}+v_{1}^{2}+\sqrt{v_{1}^{4}+4 z_{1} w_{1} v_{1}^{2}-4 v_{1}^{2} w_{1}^{2}}}{2\left(z_{1}^{2}+v_{1}^{2}\right)}} & \widehat{x} \leq \bar{x}, \\
\hat{x} & g_{1}(\widehat{x}) \geq g_{2}(\widehat{x}) \\
\sqrt{\frac{2 z_{2} w_{2}+v_{2}^{2}+\sqrt{v_{2}^{4}+4 z_{2} w_{2} v_{2}^{2}-4 v_{2}^{2} w_{2}^{2}}}{2\left(z_{2}^{2}+v_{2}^{2}\right)}} & \widehat{x}>\bar{x}(\bar{x})<g_{2}(\widehat{x}) \\
& g_{1}(\widehat{x}) \geq g_{2}(\widehat{x})
\end{array}\right.
$$

where $w_{2}=\left(B_{4}-A_{1}\right) c^{2}, z_{2}=\left(A_{1}-B_{4}\right)\left(b^{2}-c^{2}\right)-B_{3} a^{2}$, and $v_{2}=2\left(B_{4}-A_{1}\right) b c$.

Proof Note that $B_{3}+B_{4}=\frac{A_{1}\left(B_{1}+B_{2}\right)}{A_{1}+B_{1}+B_{2}} \leq A_{1}$, hence we have

$$
\begin{aligned}
& B_{3} a^{2} \bar{x}^{2}+B_{4}\left(b \bar{x}+c \sqrt{1-\bar{x}^{2}}\right)^{2} \\
= & B_{3} a^{2} \bar{x}^{2}+B_{4} a^{2} \bar{x}^{2} \leq A_{1} a^{2} \bar{x}^{2}
\end{aligned}
$$

Equation (28) indicates that the conclusion of $g_{1}(\bar{x}) \leq$ $g_{2}(\bar{x})$ must be hold. Then, Case 2 would only be divided into four scenarios:

1. In this scenario, $\widehat{x} \leq \bar{x}$ and $g_{1}(\widehat{x}) \geq g_{2} \widehat{x}$. There is only one cross point between $g_{1}(x)$ and $A_{1} a^{2} x^{2}$, which occurs after the point $\widehat{x}$. Hence,

$\min \left(g_{1}(x), g_{2}(x)\right)$ achieves its maximal value when

$B_{3} a^{2} x^{2}+B_{4}\left(b x+c \sqrt{1-x^{2}}\right)^{2}=A_{1} a^{2} x^{2}$, i.e.,

$x^{*}=\sqrt{\frac{2 z_{1} w_{1}+v_{1}^{2}+\sqrt{v_{1}^{4}+4 z_{1} w_{1} v_{1}^{2}-4 v_{1}^{2} w_{1}^{2}}}{2\left(z_{1}^{2}+v_{1}^{2}\right)}}$.

2. In this scenario, $\widehat{x} \leq \bar{x}$ and $g_{1}(\widehat{x})<g_{2}(\widehat{x})$. And $g_{1}(x)$ and $A_{1} a^{2} x^{2}$ also has one cross point, which occurs 
before the point at which $g_{1}(x)$ is maximized. Hence, $\min \left(g_{1}(x), g_{2}(x)\right)$ achieves its maximal value at the point when $g_{1}(x)$ is maximized, i.e., $x^{*}=\widehat{x}$.

3. In this scenario, $\widehat{x}>\bar{x}$ and $g_{1}(\widehat{x}) \geq g_{2}(\widehat{x})$. There may be one or two cross points between $g_{1}(x)$ and $A_{1}\left(b x+c \sqrt{1-x^{2}}\right)^{2}$, and the bigger one would be located in the interval $(\bar{x}, \widehat{x})$. Hence, the optimal $x$ is the bigger root of $B_{3} a^{2} x^{2}+B_{4}\left(b x+c \sqrt{1-x^{2}}\right)^{2}=$ $A_{1}\left(b x+c \sqrt{1-x^{2}}\right)^{2}$, i.e., $x^{*}=\sqrt{\frac{2 z_{2} w_{2}+v_{2}^{2}+\sqrt{v_{2}^{4}+4 z_{2} w_{2} v_{2}^{2}-4 v_{2}^{2} w_{2}^{2}}}{2\left(z_{2}^{2}+v_{2}^{2}\right)}}$.

4. In this scenario, $\widehat{x}>\bar{x}$ and $g_{1}(\widehat{x})<g_{2}(\widehat{x})$. Obviously, the optimal $x$ is $\widehat{x}$.

\subsection{Case 3: $a \geq \frac{b^{2}+c^{2}}{b}$}

In this case, $g_{2}(x)$ is also a piecewise function, as shown by the blue line in Fig. 4c. However, the maximum of $g_{2}(x)$ is obtained at $\bar{x}=\frac{b}{\sqrt{b^{2}+c^{2}}}$, i.e., the maximum point of the function $A_{1}\left(b x+c \sqrt{1-x^{2}}\right)^{2}$.

Lemma 3 When $a \geq \frac{b^{2}+c^{2}}{b}$, the optimal solution is expressed as

$$
x^{*}=\left\{\begin{array}{cl}
\bar{x} & g_{1}(\bar{x}) \geq g_{2}(\bar{x}) \\
\sqrt{\frac{\left.2 z_{2} w_{2}+v_{2}^{2}+\sqrt{v_{2}^{4}+4 z_{2} w_{2} v_{2}^{2}-4 v_{2}^{2} w_{2}^{2}}\right)}{2\left(z_{2}^{2}+v_{2}^{2}\right)}} & g_{1}(\bar{x})<g_{2}(\bar{x}), \\
\widehat{x} & g_{1}(\widehat{x}) \geq g_{2}(\widehat{x}) \\
& g_{1}(\widehat{x})<g_{2}(\widehat{x})
\end{array}\right.
$$

Proof Recall that $B_{3} a^{2} x^{2}$ increases monotonically when $x \in[0,1]$ and $B_{4}\left(b x+c \sqrt{1-x^{2}}\right)^{2}$ increases in the interval $[0, \bar{x}]$ and then decreases in the interval $(\bar{x}, 1]$. Due to the fact that $g_{1}(x)$ is a linear combination of these two functions and the two coefficients $B_{3}$ and $B_{4}$ are positive. Hence, we can claim that the maximum of $g_{1}(x)$ must be obtained when $x>\bar{x}$. Then, Case 3 would only be divided into three scenarios:

1. In this scenario, $g_{1}(\bar{x}) \geq g_{2}(\bar{x})$. There may be one or two cross points between $g_{1}(x)$ and

$A_{1}\left(b x+c \sqrt{1-x^{2}}\right)^{2}$, but which is(are) before the point at which $g_{2}(x)$ is maximized. Hence, the optimal $x$ is $\bar{x}$.

2. In this scenario, $g_{1}(\bar{x})<g_{2}(\bar{x})$ and $g_{1}(\widehat{x}) \geq g_{2}(\widehat{x})$. The optimal solution is obtained at the point making

$$
\begin{aligned}
& B_{3} a^{2} x^{2}+B_{4}\left(b x+c \sqrt{1-x^{2}}\right)^{2}= \\
& A_{1}\left(b x+c \sqrt{1-x^{2}}\right)^{2} \text { and is the bigger root of this } \\
& \text { equation, i.e., } x^{*}=\sqrt{\frac{2 z_{2} w_{2}+v_{2}^{2}+\sqrt{v_{2}^{4}+4 z_{2} w_{2} v_{2}^{2}-4 v_{2}^{2} w_{2}^{2}}}{2\left(z_{2}^{2}+v_{2}^{2}\right)}} .
\end{aligned}
$$

3. In this scenario, $g_{1}(\widehat{x})<g_{2}(\widehat{x})$. Obviously, the optimal $x$ is $\widehat{x}$.

Having obtained $x^{*}$, i.e., the optimal beamforming vector $\mathbf{w}_{2}^{\text {opt }}$, the optimal power splitting ratio at $R_{i}$ is given by

$$
\lambda_{i}^{*}=1-\frac{\min \left\{\begin{array}{l}
B_{3}\left|\mathbf{h}_{S R_{1}}^{T} \mathbf{w}_{2}^{o p t}\right|^{2}+B_{4}\left|\mathbf{h}_{S R_{2}}^{T} \mathbf{w}_{2}^{o p t}\right|^{2}, \\
\min \left(A_{1}\left|\mathbf{h}_{S R_{1}}^{T} \mathbf{w}_{2}^{o p t}\right|^{2}, A_{1}\left|\mathbf{h}_{S R_{2}}^{T} \mathbf{w}_{2}^{o p t}\right|^{2}\right)
\end{array}\right\}}{A_{1}\left|\mathbf{h}_{S R_{i}}^{T} \mathbf{w}_{2}^{o p t}\right|^{2}}
$$

Remark 2 For the PSR protocol, $\gamma_{D F, 1}$ and $\gamma_{D F, 2}$ could be balanced as far as possible, but the efficient information transmission time is set to be a constant. The drawback of this setting is explained as follows: the SNR of the second hop is subjected to twice channel fading and the fixed information transmission time (T/2) would result in a small efficient SNR, which is the main factor affecting the network throughput. However, as we will see, in the HR protocol, the information transmission time can be adjusted flexibly.

\section{Hybrid relaying (HR) protocol}

As shown in Fig. 5, the HR protocol is a combination of the TSR protocol and the PSR protocol. The transmission process of the HR protocol and the TSR protocol is similar. However, in the second phase, a fraction $\left(\lambda_{i} \in[0,1]\right.$, $i \in\{1,2\})$ of the received signals would be used for energy harvesting at $R_{i}$, and a fraction $\left(1-\lambda_{i}\right)$ of the received signal would be used for information reception at $R_{i}$.

Hence, the received SNR at $R_{i}$ after the second phase is given in (13) and the SNR of the first hop is given in (14).

Observe that the harvested energy by $R_{i}$ in the HR protocol comes from the first phase and the second phase. Therefore, the harvested energy by $R_{i}$ after the second phase is given as

$$
E_{R_{i}}=\frac{\eta \alpha P_{S}\left|\mathbf{h}_{S R_{i}}^{T} \mathbf{w}_{1}\right|^{2}}{d_{S R}^{m}}+\frac{\eta \frac{1-\alpha}{2} P_{S}\left|\mathbf{h}_{S R_{i}}^{T} \mathbf{w}_{2}\right|^{2} \lambda_{i}}{d_{S R}^{m}}
$$

then the SNR of the second hop is expressed as 


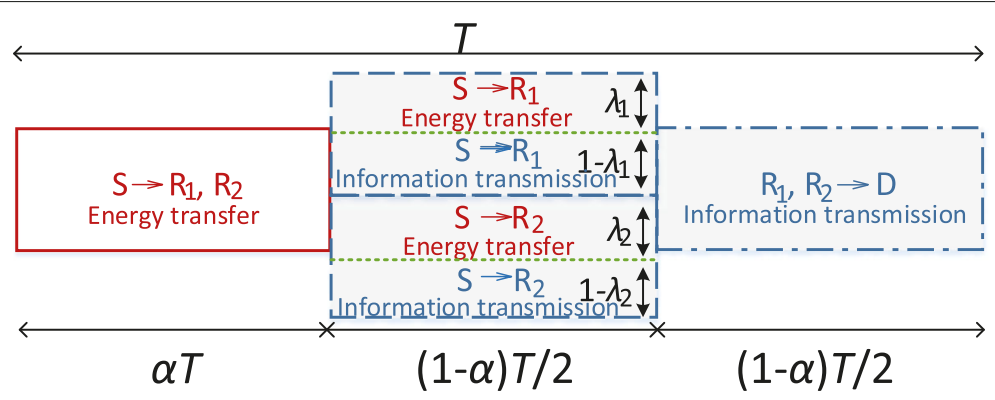

Fig. 5 Diagram of the HR protocol

$$
\begin{aligned}
\gamma_{D F, 2}= & \frac{P_{R_{1}}\left|f_{R_{1} D}\right|^{2}}{d_{R D}^{m} N_{0}}+\frac{P_{R_{2}}\left|f_{R_{2} D}\right|^{2}}{d_{R D}^{m} N_{0}} \\
= & \frac{E_{R_{1}}\left|f_{R_{1} D}\right|^{2}}{d_{R D}^{m} N_{0}(1-\alpha) / 2}+\frac{E_{R_{2}}\left|f_{R_{2} D}\right|^{2}}{d_{R D}^{m} N_{0}(1-\alpha) / 2} \\
= & \frac{\alpha}{1-\alpha}\left(A_{2}\left|\mathbf{h}_{S R_{1}}^{T} \mathbf{w}_{1}\right|^{2}+A_{3}\left|\mathbf{h}_{S R_{2}}^{T} \mathbf{w}_{1}\right|^{2}\right) \\
& +B_{1}\left|\mathbf{h}_{S R_{1}}^{T} \mathbf{w}_{2}\right|^{2} \lambda_{1}+B_{2}\left|\mathbf{h}_{S R_{2}}^{T} \mathbf{w}_{2}\right|^{2} \lambda_{2}
\end{aligned}
$$

Interestingly, (32) can be regarded as the sum of (4) and (21), i.e., the SNR of the second hop under the HR protocol can be understood as the sum of the SNR of the second hop under the TSR and the PSR protocols.

Therefore, the achievable end-to-end throughput is given by

$$
R_{D F}=\frac{1-\alpha}{2} \log _{2}\left(1+\min \left(\gamma_{D F, 1}, \gamma_{D F, 2}\right)\right)
$$

Next, we aim to maximize $R_{D F}$ by optimizing $\alpha, \lambda_{1}, \lambda_{2}$, $\mathbf{w}_{1}$ and $\mathbf{w}_{2}$. The optimization problem can be formulated as

$$
\begin{aligned}
& \text { P4: } \max _{\alpha, \lambda_{1}, \lambda_{2}, \mathbf{w}_{1}, \mathbf{w}_{2}} R_{D F} \\
& \text { s.t. } \quad \alpha, \lambda_{1}, \lambda_{2} \in[0,1],\left\|\mathbf{w}_{1}\right\|^{2}=\left\|\mathbf{w}_{2}\right\|^{2}=1 .
\end{aligned}
$$

Same as the TSR protocol, the optimal $\alpha$ must be smaller than or equal to $\frac{f_{1}\left(x_{1}^{*}\right)}{f_{1}\left(x_{1}^{*}\right)+f_{2}\left(x_{2}^{*}\right)}$; otherwise, there is no necessity to split a fraction of the received signal at each relay as additional harvested energy in the second phase. And when the possible $\alpha$ is fixed, the optimal solution $\left(\lambda_{1}^{*}, \lambda_{2}^{*}\right.$, $\mathbf{w}_{1}^{\text {opt }}, \mathbf{w}_{2}^{\text {opt }}$ ) must happen at $\gamma_{D F, 1}=\gamma_{D F, 2}$. Moreover, the received SNR at $R_{1}$ and $R_{2}$ should be same. Hence, we have

$$
\begin{aligned}
\gamma_{\mathrm{ete}}= & A_{1}\left|\mathbf{h}_{S R_{1}}^{T} \mathbf{w}_{2}\right|^{2}\left(1-\lambda_{1}\right)=A_{1}\left|\mathbf{h}_{S R_{2}}^{T} \mathbf{w}_{2}\right|^{2}\left(1-\lambda_{2}\right) \\
= & \frac{\alpha}{1-\alpha}\left(A_{2}\left|\mathbf{h}_{S R_{1}}^{T} \mathbf{w}_{1}\right|^{2}+A_{3}\left|\mathbf{h}_{S R_{2}}^{T} \mathbf{w}_{1}\right|^{2}\right) \\
& +B_{1}\left|\mathbf{h}_{S R_{1}}^{T} \mathbf{w}_{2}\right|^{2} \lambda_{1}+B_{2}\left|\mathbf{h}_{S R_{2}}^{T} \mathbf{w}_{2}\right|^{2} \lambda_{2}
\end{aligned}
$$

Based on (35), $\gamma_{\text {ete }}$ is given by

$$
\begin{aligned}
\gamma_{\mathrm{ete}}= & \frac{\frac{\alpha}{1-\alpha}\left(A_{2}\left|\mathbf{h}_{S R_{1}}^{T} \mathbf{w}_{1}\right|^{2}+A_{3}\left|\mathbf{h}_{S R_{2}}^{T} \mathbf{w}_{1}\right|^{2}\right)}{1+\frac{B_{1}}{A_{1}}+\frac{B_{2}}{A_{1}}} \\
& +\frac{B_{1}\left|\mathbf{h}_{S R_{1}}^{T} \mathbf{w}_{2}\right|^{2}+B_{2}\left|\mathbf{h}_{S R_{2}}^{T} \mathbf{w}_{2}\right|^{2}}{1+\frac{B_{1}}{A_{1}}+\frac{B_{2}}{A_{1}}} \\
= & \frac{\alpha}{1-\alpha}\left(B_{5}\left|\mathbf{h}_{S R_{1}}^{T} \mathbf{w}_{1}\right|^{2}+B_{6}\left|\mathbf{h}_{S R_{2}}^{T} \mathbf{w}_{1}\right|^{2}\right) \\
& +B_{3}\left|\mathbf{h}_{S R_{1}}^{T} \mathbf{w}_{2}\right|^{2}+B_{4}\left|\mathbf{h}_{S R_{2}}^{T} \mathbf{w}_{2}\right|^{2}
\end{aligned}
$$

where $B_{5}=\frac{A_{2}}{1+\frac{B_{1}}{A_{1}}+\frac{B_{2}}{A_{1}}}$ and $B_{6}=\frac{A_{3}}{1+\frac{B_{1}}{A_{1}}+\frac{B_{2}}{A_{1}}}$.

Combining the constraint in (34), the efficient SNR is given by

$$
\begin{aligned}
\gamma_{\mathrm{eff}}=\min & \left(\frac{\alpha}{1-\alpha}\left(B_{5}\left|\mathbf{h}_{S R_{1}}^{T} \mathbf{w}_{1}\right|^{2}+B_{6}\left|\mathbf{h}_{S R_{2}}^{T} \mathbf{w}_{1}\right|^{2}\right)\right. \\
& +B_{3}\left|\mathbf{h}_{S R_{1}}^{T} \mathbf{w}_{2}\right|^{2}+B_{4}\left|\mathbf{h}_{S R_{2}}^{T} \mathbf{w}_{2}\right|^{2} \\
& \left.\min \left(A_{1}\left|\mathbf{h}_{S R_{1}}^{T} \mathbf{w}_{2}\right|^{2}, A_{1}\left|\mathbf{h}_{S R_{2}}^{T} \mathbf{w}_{2}\right|^{2}\right)\right)
\end{aligned}
$$

Based on (37), we can see that the optimization process for $\mathbf{w}_{1}$ and $\mathbf{w}_{2}$ will be independent. For a fixed $\alpha$, the optimal beamforming vectors $\mathbf{w}_{1}^{\text {opt }}$ and $\mathbf{w}_{2}^{\text {opt }}$ for maximizing $\gamma_{\text {eff }}$ admit the same form in (7). And $\mathbf{w}_{1}^{\text {opt }}$ can be obtained using the same method for solving P1; here, we omit the computing process. Denote $Z=B_{5}\left|\mathbf{h}_{S R_{1}}^{T} \mathbf{w}_{1}^{\text {opt }}\right|^{2}+$ $B_{6}\left|\mathbf{h}_{S R_{2}}^{T} \mathbf{w}_{1}^{\text {opt }}\right|^{2}$, then (37) is rewritten as

$$
\begin{aligned}
\gamma_{\mathrm{eff}}=\min & \left(\frac{\alpha Z}{1-\alpha}+B_{3}\left|\mathbf{h}_{S R_{1}}^{T} \mathbf{w}_{2}\right|^{2}+B_{4}\left|\mathbf{h}_{S R_{2}}^{T} \mathbf{w}_{2}\right|^{2}\right. \\
& \left.\min \left(A_{1}\left|\mathbf{h}_{S R_{1}}^{T} \mathbf{w}_{2}\right|^{2}, A_{1}\left|\mathbf{h}_{S R_{2}}^{T} \mathbf{w}_{2}\right|^{2}\right)\right)
\end{aligned}
$$


Now, we can observe that (38) admits the same form shown in (22). Hence, P4 can be reformulated as

$$
\begin{aligned}
& \text { P4: } \max _{\alpha, x} \frac{1-\alpha}{2} \log _{2}\left(1+\min \left(\frac{\alpha Z}{1-\alpha}+g_{1}(x), g_{2}(x)\right)\right) \\
& \text { s.t. } \alpha \in[0,1], x \in[0,1] \text {. }
\end{aligned}
$$

Due to the fact that $\alpha$ is coupled with $x$ and problem P4 is non-convex, finding the optimal solution of $\alpha$ and $x$ is difficult. However, we can pursue a suboptimal design by adopting the idea of alternating optimization, which has been shown to achieve a decent performance [48].

Specifically, observing (39), we can see that, when $\alpha$ is fixed, the closed-form solution of the optimal $x$ (denote as $x_{\alpha}^{*}$ ) can be obtained based on the analytical process in Section 4 . When $x$ is fixed, by employing the same method for solving P1, the closed-form solution of the optimal $\alpha$ (denote as $\alpha_{x}^{*}$ ) can be obtained as

$$
\begin{aligned}
\alpha_{x}^{*}= & \min \left(\max \left(\frac{g_{2}(x)-g_{1}(x)}{g_{2}(x)-g_{1}(x)+Z}, 0\right),\right. \\
& \left.\frac{e^{\mathbb{W}\left(\frac{Z-1-g_{1}(x)}{e}\right)+1}-1}{Z+e^{\mathbb{W}\left(\frac{Z-1-g_{1}(x)}{e}\right)+1}-1}\right)
\end{aligned}
$$

The proposed alternating optimization approach is summarized in Algorithm 1. In particular, since the objective function has a finite value and the alternating optimization design is nondecreasing in each step, Algorithm 1 is guaranteed to be convergent. In Section 7, we will show the proposed alternating optimization approach achieves near-optimal performance. Moreover, given the required precision of the solution $\sigma$, Algorithm 1 only requires $\log \left(\frac{1}{\sigma}\right)$ iterations, while conventional one dimensional search would require $\frac{1}{\sigma}$ iterations.

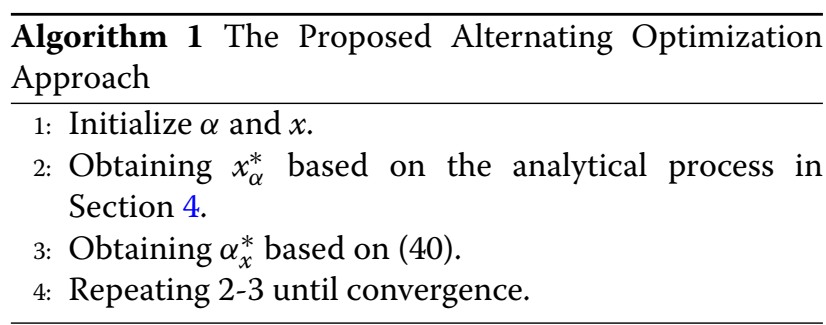

Finally, based on the known $\alpha^{*}$, the beamforming vectors $\mathbf{w}_{1}^{\text {opt }}$ and $\mathbf{w}_{2}^{\text {opt }}$, the optimal power splitting ratio at $R_{i}$ is given by

$$
\begin{aligned}
& \lambda_{i}^{*}=1- \\
& \frac{\min \left\{\begin{array}{l}
\frac{\alpha^{*} Z}{1-\alpha^{*}}+B_{3}\left|\mathbf{h}_{S R_{1}}^{T} \mathbf{w}_{2}^{\mathrm{opt}}\right|^{2}+B_{4}\left|\mathbf{h}_{S R_{2}}^{T} \mathbf{w}_{2}^{\mathrm{opt}}\right|^{2}, \\
\min \left(A_{1}\left|\mathbf{h}_{S R_{1}}^{T} \mathbf{w}_{2}^{\mathrm{opt}}\right|^{2}, A_{1}\left|\mathbf{h}_{S R_{2}}^{T} \mathbf{w}_{2}^{\mathrm{opt}}\right|^{2}\right)
\end{array}\right.}{A_{1}\left|\mathbf{h}_{S R_{i}}^{T} \mathbf{w}_{2}^{\mathrm{opt}}\right|^{2}}
\end{aligned}
$$

Remark 3 The HR protocol combines the advantages of the TSR and PSR protocols and achieves the best tradeoff relationship between the information transmission time and the efficient SNR. Hence, under the proposed network architecture, it can be regarded as the optimal protocol in term of maximizing the network throughput.

\section{Simulation results}

In this section, numerical results are presented to compare the throughput performance of the TSR, PSR, and HR protocols in terms of some key parameters. And insights on the optimal TS and PS ratios are also provided. In all simulations, the distance between the source and the destination is set to 10 meters $(\mathrm{m})$, the path loss factor is equal to 3 , and the energy conversion efficiency is equal to 0.6 . For all figures, each point is obtained by averaging $2 \times 10^{4}$ independent and random simulations. The channel model has been introduced in Section 3. The number of antennas equipped at the source is set to $N=3$ and $N=8$.

\subsection{Throughput performance comparison}

To illustrate the superior performance achieved by our proposed design scheme, we further plot the performance curves of another two schemes:

- One scheme is called "Beamforming towards the best relay" (BTBR1). In particular, for BTBR1, the beamforming vector $\mathbf{w}_{i}(i \in\{1,2\})$ would be set to $\frac{\mathbf{h}_{S R_{b}}^{*}}{\left\|\mathbf{h}_{S R_{b}}\right\|}$, where $b=\arg \max _{i \in\{1,2\}} \min \left\|\mathbf{h}_{S R_{i}}\right\|\left|f_{R_{i} D}\right|$.

- The other scheme is called BTBR2. In particular, for BTBR2, $\mathbf{w}_{i}(\forall i \in\{1,2\})$ is also set to $\frac{\mathbf{h}_{S R_{b}}^{*}}{\left\|\mathbf{h}_{S R_{b}}\right\|}$ under these three protocols. However, the relay $R_{\{1,2\} / b}$ does not participant in the transmission process.

Figures 6 and 7 compare the throughput performance of the three protocols under our proposed schemes, BTBR1 and BTBR2, in terms of different transmit SNR and $N$. Note that the transmit SNR is specified by $P_{S} / N_{0}$. First, it is obvious that our proposed scheme outperforms another two schemes, which is mainly resulted from the optimal beamforming design provided in our scheme. For BTBR1, the received SNR at $D$ from $R_{1}$ and $R_{2}$ would 


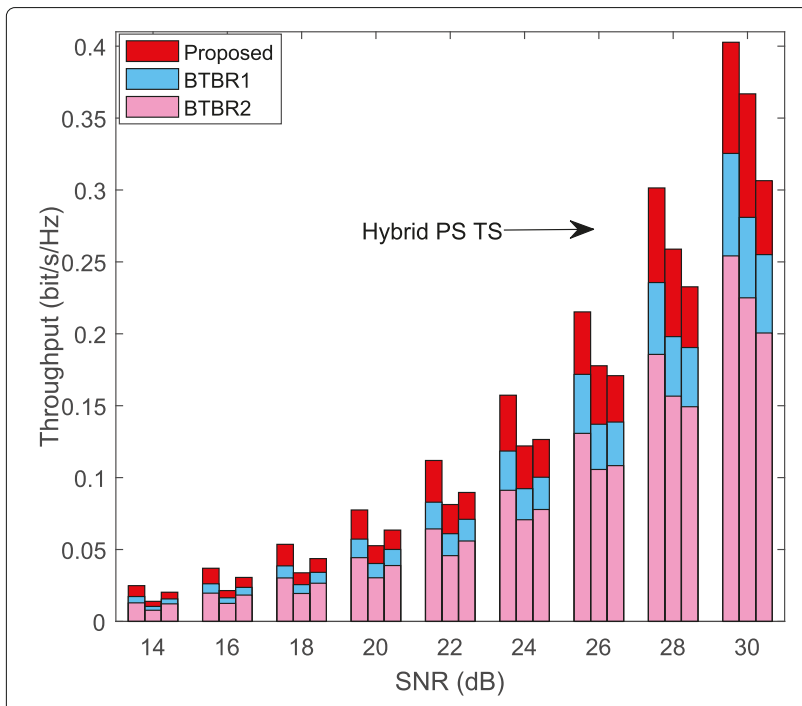

Fig. 6 Throughput performance of different protocols versus transmit $\mathrm{SNR}, d_{S R}=2 \mathrm{~m}, \mathrm{~N}=3$

have certain difference, this phenomenon limits its performance. The performance of BTBR2 will be further deteriorate because of the inadequate utilization for the relay nodes. Second, the throughput performance achieved by each protocol increases with the increasing SNR. This phenomenon is obvious, as the larger transmit power not only contributes more harvested energy at each relay, but also provides higher channel capacity of the first hop. Third, by equipping larger number of antennas at the source, the energy transfer efficiency can be boosted up and the higher spatial diversity can be obtained.

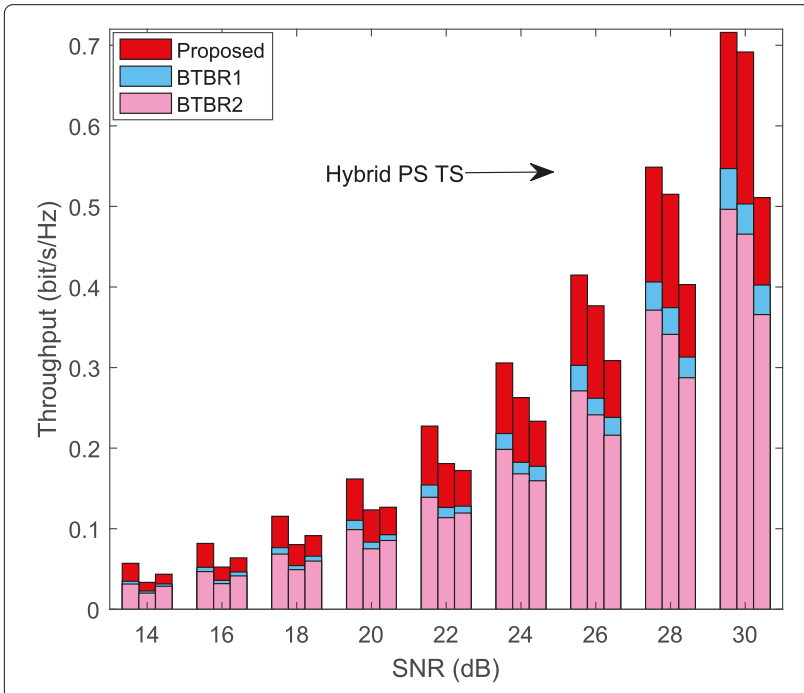

Fig. 7 Throughput performance of different protocols versus transmit $\mathrm{SNR}, d_{S R}=2 \mathrm{~m}, N=8$
Hence, the network performance can be improved greatly.

Now, let us formally compare these three protocols. As we can see, (i) the TSR protocol outperforms the PSR protocol in the low SNR region (such as $14-24 \mathrm{~dB}$ and $14-20 \mathrm{~dB}$ in our proposed scheme when $N=3$ and $N=8$ ), and the latter outperforms the former in the high SNR region (such as $26-30 \mathrm{~dB}$ and $22-30 \mathrm{~dB}$ in our proposed scheme when $N=3$ and $N=8$ ); and (ii) the HR protocol owns the best performance in any SNR region. The corresponding reasons are given as follows:

(i) The values of the throughput shown in (5), (16), and (33) depend on the information transmission time and the channel capacities of the two hops. In the low SNR region, the main factor affecting the throughput is the channel capacities of the two hops, the values of which would increase rapidly by expanding the time length of the first phase in the TSR protocol. This is because that the logarithmic function has large slope when the independent variable is in a small range. However, in the PSR protocol, the channel capacities cannot be further enhanced after implementing optimal power splitting scheme at each relay. In the high SNR region, as the logarithmic function grows slowly, the channel capacities would change more stably and the throughput is mainly related to the information transmission time, the value of which is maximized in the PSR protocol.

(ii) The reasons are presented in Remarks 1, 2 ,and 3; here, we omit the explaining process.

Figures 8 and 9 compare the throughput performance of the three protocols under our proposed scheme ${ }^{4}$ in terms of the different relays' locations. In particular, the curves associated with the global optimization design about the HR protocol are acquired via a two-dimensional search over $\alpha$ and $x$. First, it can be readily observed that the alternating optimization design achieves almost the same performance as the global optimization design. Second, once again, the respective advantages of the TSR protocol and the PSR protocol in the low SNR (20dB) and high SNR (30dB) regions are presented in Figs. 8 and 9 , respectively. Third, it is obvious that the throughput achieved by these three protocols own worst performance when the two relays are located at the middle of the source and the destination. Here, we give the detailed reasons: for these three protocols, the efficient SNR is determined by the second hop with great probability, as the SNR of the second hop suffers from twice channel fading. In the expression of $\gamma_{D F, 2}$, we can see that $d_{S R}^{m} d_{R D}^{m}$ in the denominator is the primary item affecting the

\footnotetext{
${ }^{4}$ Since we have presented BTBR1 and BTBR2 in Figs. 6 and 7 and shown their poor performance, we only present our proposed scheme in the following figures.
} 


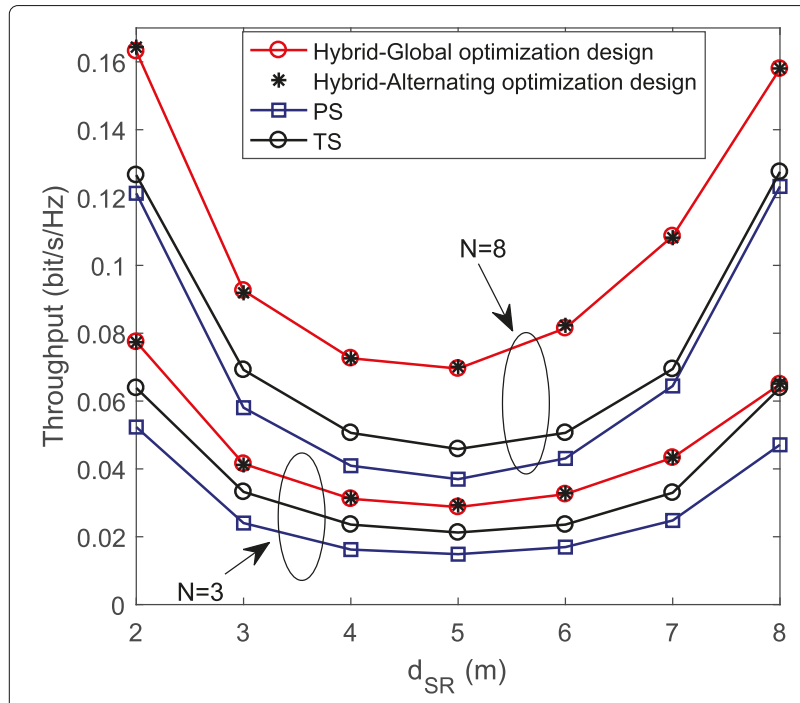

Fig. 8 Throughput performance of different protocols versus $d_{S R}, S N R$ $=20 \mathrm{~dB}$

value of the SNR. And the value of $d_{S R}^{m} d_{R D}^{m}$ is maximized when $d_{S R}=d_{R D}=5 \mathrm{~m}$. The approximate symmetry of the Figs. 8 and 9 can be explained by our given reason.

\subsection{Insights on the optimal TS, PS, and TS\&PS ratios}

In this subsection, we provide some insights on the optimal TS ratio $\alpha$ and PS ratio $\lambda$ of these three protocols under our proposed scheme. Specially, the optimal $\lambda$ is specified by the average value of $\lambda_{1}^{*}$ and $\lambda_{2}^{*}$ in the PSR and HR protocols.

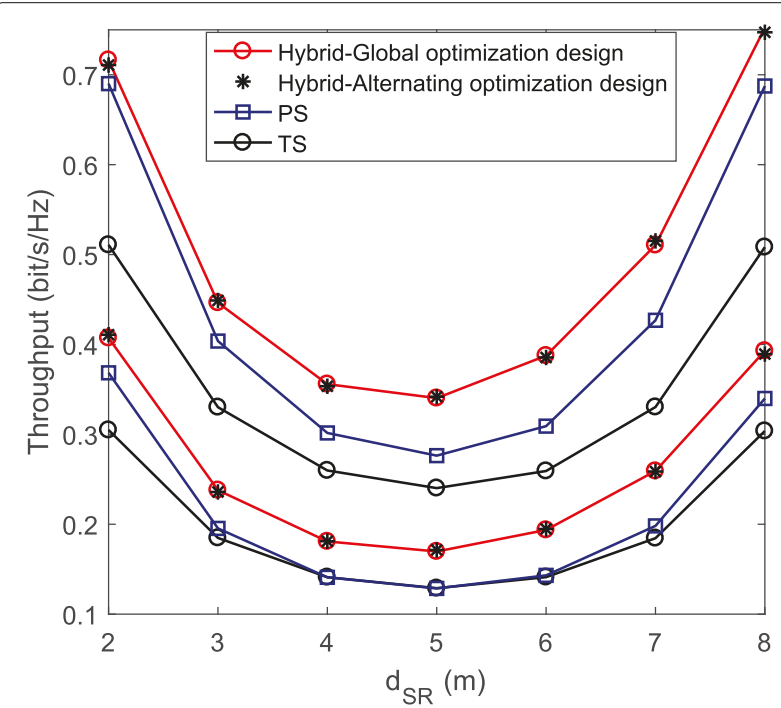

Fig. 9 Throughput performance of different protocols versus $d_{S R}$, SNR $=30 \mathrm{~dB}$
In Fig. 10, we can see that, with the increasing transmit power, (i) the optimal TS ratio (denote as $\alpha_{T S R}^{*}$ ) of the TSR protocol decreases linearly, (ii) the optimal PS ratio (denote as $\lambda_{P S R}^{*}$ ) of the PSR protocol is unchanged and approaches 1, and (iii) the optimal PS ratio (denote as $\lambda_{H R}^{*}$ ) of the HR protocol increases and is close to $\lambda_{P S R}^{*}$ gradually; the optimal TS ratio (denote as $\alpha_{H R}^{*}$ ) of the HR protocol decreases and is always smaller than $\alpha_{T S R}^{*}$. The corresponding reasons are given as follows:

(i) As mentioned in Subsection 7.1, with the increasing transmit power, the channel capacities of the two hops would change slowly. Then, a larger information transmission time would contribute the larger throughput.

(ii) The purpose of power splitting in the PSR protocol is to balance the capacities of the two hops as much as possible. No matter what the transmit power is, once other parameters are fixed, the ratio allocation would not be changed. And the large $\lambda_{P S R}^{*}$ is the result of the twice fading suffered by the SNR of the second hop.

(iii) For the HR protocol, $\alpha_{H R}^{*}$ must be smaller than $\alpha_{T S R}^{*}$, as each relay can split some fraction of the received signal at the second phase for additional harvested energy to increase the channel capacity of the second hop. Obviously, as $\alpha_{H R}^{*}$ decreases, $\lambda_{H R}^{*}$ would increase accordingly so that the best balance can be achieved. Finally, the HR protocol would completely evolve into the PSR protocol when the transmit SNR approaches infinity.

In Fig. 11, we can see that, with the increasing $d_{S R}$, (i) $\lambda_{P S R}^{*}$ and $\lambda_{H R}^{*}$ decrease little by little and (ii) $\alpha_{T S R}^{*}$ and $\alpha_{H R}^{*}$ increase first and then decrease. The corresponding reasons are given as follows:

(i) With the increasing $d_{S R}$, the channel capacities of the first hop and the second hop would reduce and increase, respectively. Hence, although the capacity of the second hop is suffered from twice channel fading, the relays only need smaller power to compensate it.

(ii) For the TSR protocol and the HR protocol, the channel capacity of the second hop would get its minimum when the relays are located at the middle of the source and the destination, which needs to be enhanced by increasing $\alpha$.

\section{Conclusion and future work}

In this paper, we have investigated the throughput performance of three relaying protocols, i.e., the TSR protocol, the PSR protocol, and the HR protocol for the wireless-powered multi-antenna dual-relay network. To maximize the throughput of each protocol, we have provided the detailed design processes for the optimal energy and information beamforming vectors at the multi-antenna source, the optimal time switching, and power splitting ratios. Based on theoretical analysis and 


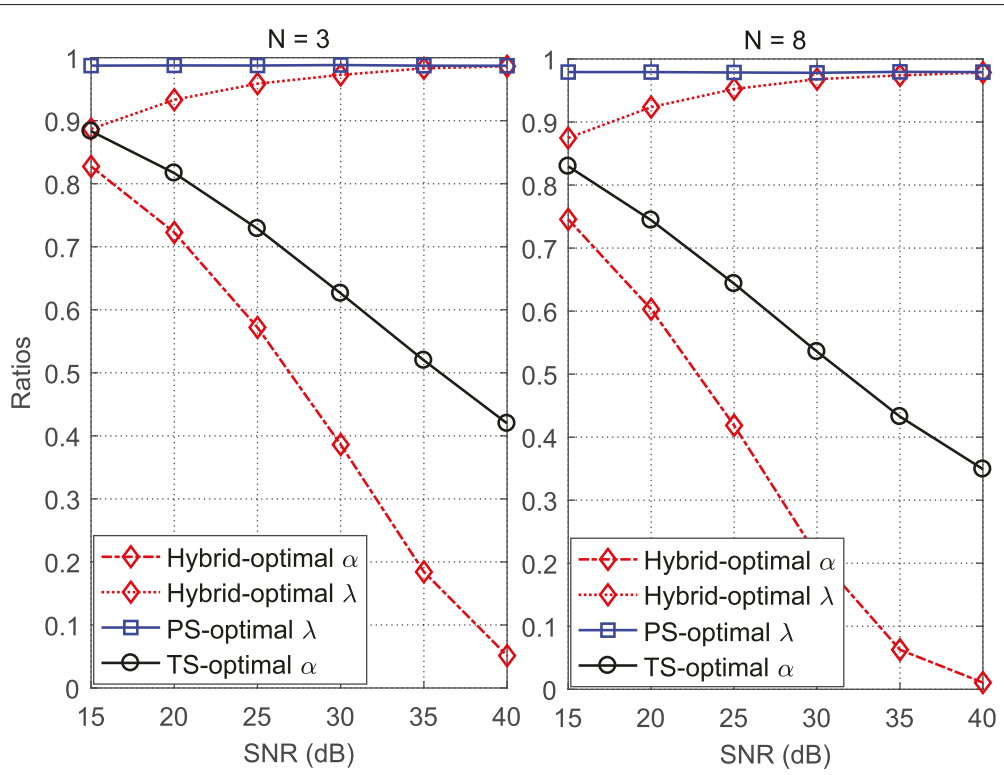

Fig. 10 Optimal $\alpha$ and $\lambda$ under different protocols versus transmit SNR, $d_{S R}=2 \mathrm{~m}$

simulation results, we have found that the TSR protocol and the PSR protocol have their respective advantages in the low and high SNR regions. And the best performance can be achieved by employing the HR protocol and setting the relays closer to the source or the destination concurrently.

For future works, we will consider a more challenging scenario such that there exist any number of relays and each relay works in the FD mode. Specifically, the FD strategy of each relay enables the simultaneous transmission/reception of information and energy signals. Then, it is conceivable that these would significantly change the methods of designing energy and information beamformers at the source and the detailed optimization process for finding the optimal time switching and power splitting ratios.
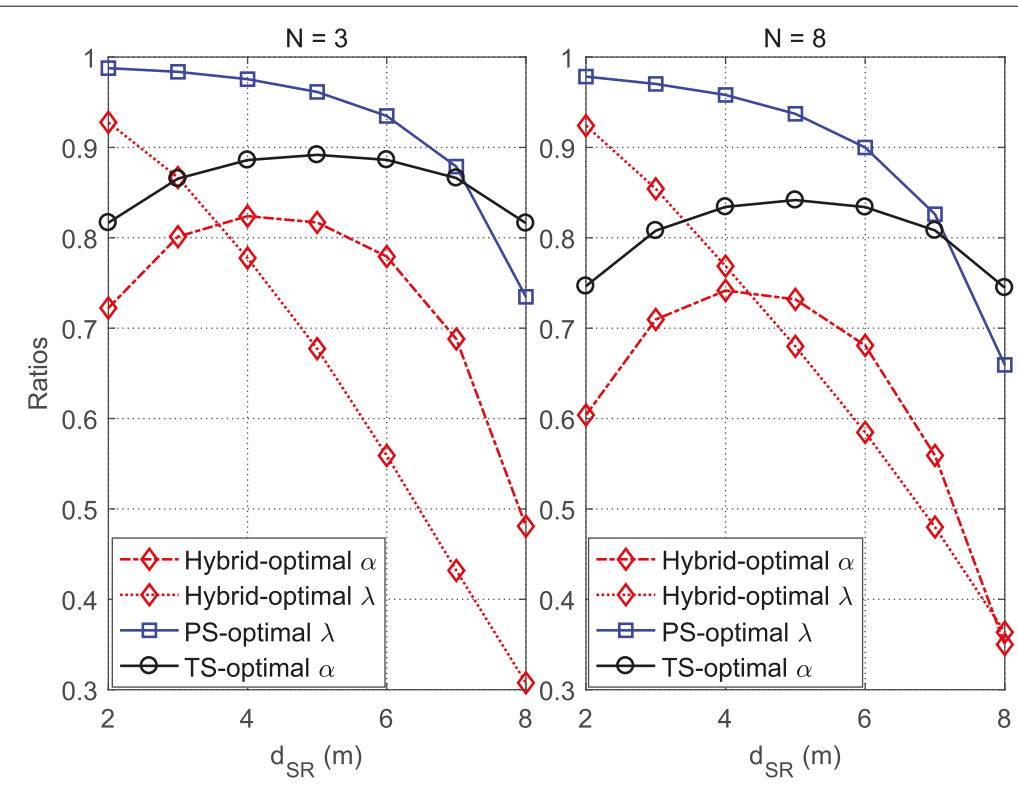

Fig. 11 Optimal $\alpha$ and $\lambda$ under different protocols versus $d_{S R}, S N R=20 d B$ 


\section{Abbreviations}

CCl: Co-channel interference; CSI: Channel state information; DF: Decode-and-forward; DSTC: Distributed space-time coding; FD: Full-duplex; HD: Half-duplex; HR: Hybrid relaying; HTT: Harvest-then-transmit; IOTs: Internet of Things; MIMO: Multiple-input-multi-output; NOMA: Nonorthogonal multiple access; OFDM: Orthogonal frequency-division multiplexing; PSR: Power splitting relaying; QoS: Quality of Service; RF: Radio frequency; SDP: Semidefinite programming; SNR: Signal-to-noise ratio; TB: Transmission block; TSR: Time switching-based relaying; WET: Wireless energy transfer; WIT: Wireless information transmission; WPCN: Wireless powered communication network

\section{Acknowledgements}

This research was supported by National Natural Science Foundation of China (No. 61371122, 61471393, 61771487, and 61501507), Jiangsu Provincial Natural Science Foundation of China (BK20150719).

\section{Authors' contributions}

$\mathrm{GH}$ is the main writer of this paper. He proposed the main idea, conducted the simulations, and analyzed it. YC, LA, and XW assisted the review of this manuscript. All authors read and approved the final manuscript.

\section{Funding}

This research was supported by National Natural Science Foundation of China (No. 61371122, 61471393, 61771487, and 61501507), Jiangsu Provincial Natural Science Foundation of China (BK20150719).

\section{Availability of data and materials} Not applicable.

\section{Competing interests}

The authors declare that they have no competing interests.

\section{Received: 9 December 2018 Accepted: 17 October 2019}

\section{Published online: 05 November 2019}

\section{References}

1. F. Javed, M. K. Afzal, M. Sharif, B. Kim, Internet of Things (loT) Operating systems support, networking technologies, applications, and challenges: a comparative review. IEEE Commun. Surv. Tutorials. 20(3), 2062-2100 (2018)

2. X. Lu, P. Wang, D. Niyato, D. I. Kim, Z. Han, Wireless charging technologies: fundamentals, standards, and network applications. IEEE Commun. Surv. Tutorials. 18(2), 1413-1452 (2016)

3. S. Bi, R. Zhang, Placement Optimization of energy and information access points in wireless powered communication networks. IEEE Trans. Wirel. Commun. 15(3), 2351-2364 (2016)

4. K. W. Choi, D. I. Kim, Stochastic optimal control for wireless powered communication networks. IEEE Trans. Wirel. Commun. 15(1), 686-698 (2016)

5. Q. Wu, M. Tao, D. W. Kwan Ng, W. Chen, R. Schober, Energy-efficient resource allocation for wireless powered communication networks. IEEE Trans. Wirel. Commun. 15(3), 2312-2327 (2016)

6. K. Xiong, C. Chen, G. Qu, P. Fan, K. B. Letaief, Group cooperation with optimal resource allocation in wireless powered communication networks. IEEE Trans. Wirel. Commun. 16(6), 3840-3853 (2017)

7. E. Boshkovska, D. W. K. Ng, N. Zlatanov, A. Koelpin, R. Schober, Robust resource allocation for MIMO wireless powered communication networks based on a non-linear EH model. IEEE Trans. Commun. 65(5), 1984-1999 (2017)

8. X. Di, K. Xiong, P. Fan, H. Yang, K. B. Letaief, Optimal resource allocation in wireless powered communication networks with user cooperation. IEEE Trans. Wirel. Commun. 16(12), 7936-7949 (2017)

9. A. A. Nasir, X. Zhou, S. Durrani, R. A. Kennedy, Relaying protocols for wireless energy harvesting and information processing. IEEE Trans. Wirel. Commun. 12(7), 3622-3636 (2013)

10. S. Atapattu, J. Evans, Optimal energy harvesting protocols for wireless relay networks. IEEE Trans. Wirel. Commun. 15(8), 5789-5803 (2016)

11. R. Tao, A. Salem, K. A. Hamdi, Adaptive relaying protocol for wireless power transfer and information processing. IEEE Commun. Lett. 20(10), 2027-2030 (2016)
12. H. Lee, C. Song, S. Choi, I. Lee, Outage probability analysis and power splitter designs for SWIPT relaying systems with direct link. IEEE Commun. Lett. 21(3), 648-651 (2017)

13. D. Wang, Y. Li, Y. Ye, H. Xia, H. Zhang, in 2017 IEEE 86th Vehicular Technology Conference (VTC-Fall). Joint time allocation and power splitting schemes for DF energy harvesting relaying networks, (Toronto, 2017), pp. 1-5. https://doi.org/10.1109/vtcfall.2017.8288115

14. Y. Ye, Y. Li, D. Wang, F. Zhou, R. Q. Hu, H. Zhang, Optimal transmission schemes for DF relaying networks using SWIPT. IEEE Trans. Veh. Technol. 67(8), 7062-7072 (2018)

15. Y. Zeng, R. Zhang, Full-duplex wireless-powered relay with self-energy recycling. IEEE Wirel. Commun. Lett. 4(2), 201-204 (2015)

16. S. Luo, G. Yang, K. C. Teh, Throughput of wireless-powered relaying systems with buffer-aided hybrid relay. IEEE Trans. Wirel. Commun. 15(7), 4790-4801 (2016)

17. Y. Chen, R. Shi, W. Feng, N. Ge, AF Relaying with energy harvesting source and relay. IEEE Trans. Veh. Technol. 66(1), 874-879 (2017)

18. B. Li, Y. Rong, Joint transceiver optimization for wireless information and energy transfer in non-regenerative MIMO relay systems. IEEE Trans. Veh. Technol. https://doi.org/10.1109/tvt.2018.2846556

19. B. Li, Y. Rong, AF MIMO Relay systems with wireless powered relay node and direct link. IEEE Trans. Commun. 66(4), 1508-1519 (2018)

20. Y. Liu, Joint resource allocation in SWIPT-based multiantenna decode-and-forward relay networks. IEEE Trans. Veh. Technol. 66(10), 9192-9200 (2017)

21. R. Fan, S. Atapattu, W. Chen, Y. Zhang, J. Evans, Throughput maximization for multi-hop decode-and-forward relay network with wireless energy harvesting. IEEE Access. 6, 24582-24595 (2018)

22. Y. Gu, S. Aïssa, RF-based energy harvesting in decode-and-forward relaying systems: ergodic and outage capacities. IEEE Trans. Wirel. Commun. 14(11), 6425-6434 (2015)

23. Y. Liu, X. Wang, Information and energy cooperation in OFDM relaying: protocols and optimization. IEEE Trans. Veh. Technol. 65(7), 5088-5098 (2016)

24. M. Mohammadi, B. K. Chalise, H. A. Suraweera, C. Zhong, G. Zheng, I. Krikidis, Throughput analysis and optimization of wireless-powered multiple antenna full-duplex relay systems. IEEE Trans. Commun. 64(4), 1769-1785 (2016)

25. Y. Liu, Z. Ding, M. Elkashlan, H. V. Poor, Cooperative non-orthogonal multiple access with simultaneous wireless information and power transfer. IEEE J. Sel. Areas Commun. 34(4), 938-953 (2016)

26. Y. Xu, et al., Joint beamforming and power-splitting control in downlink cooperative SWIPT NOMA systems. IEEE Trans. Signal Proces. 65(18), 4874-4886 (2017)

27. K. Lee, J. Hong, H. Choi, M. Levorato, Adaptive wireless-powered relaying schemes with cooperative jamming for two-hop secure communication. IEEE Internet Things J. 5(4), 2793-2803 (2018)

28. Y. Dong, M. J. Hossaini, J. Cheng, V. C. M. Leung, Robust energy efficient beamforming in MISOME-SWIPT systems with proportional secrecy rate. IEEE J. Sel. Areas Commun. 37(1), 202-215 (2019)

29. H. Niu, B. Zhang, Y. Huang, D. Guo, Z. Chu, Z. Zhu, Robust secrecy beamforming and power-splitting design for multiuser MISO downlink with SWIPT. IEEE Syst. J. https://doi.org/10.1109/jsyst.2018.2819993

30. W. Mei, Z. Chen, J. Fang, Artificial noise aided energy efficiency optimization in MIMOME system With SWIPT. IEEE Commun. Lett. 21(8), 1795-1798 (2017)

31. Y. Liu, Wireless information and power transfer for multirelay-assisted cooperative communication. IEEE Commun. Lett. 20(4), 784-787 (2016)

32. Z. Ding, I. Krikidis, B. Sharif, H. V. Poor, Wireless information and power transfer in cooperative networks with spatially random relays. IEEE Trans. Wirel. Commun. 13(8), 4440-4453 (2014)

33. C. Zhang, L. Hu, Iterative dynamic power splitting for multi-relay networks with wireless energy harvesting. IEEE Signal Process. Lett. 22(12), 2274-2278 (2015)

34. M. Lin, Z. Lin, W. Zhu, J. Wang, Joint beamforming for secure communication in cognitive satellite terrestrial networks. IEEE J. Sel. Areas Commun. 36(5), 1017-1029 (2018)

35. Z. Lin, M. Lin, J. Wang, Y. Huang, W. Zhu, Robust secure beamforming for $5 \mathrm{G}$ cellular networks coexisting with satellite networks. IEEE J. Sel. Areas Commun. 36(4), 932-945 (2018) 
36. Z. Lin, M. Lin, J. Ouyang, W. Zhu, S. Chatzinotas, Beamforming for secure wireless information and power transfer in terrestrial networks coexisting with satellite networks. IEEE Signal Process. Lett. 25(8), 1166-1170 (2018)

37. Z. Lin, M. Lin, J. Wang, T. de Cola, J. Wang, Joint beamforming and power allocation for satellite-terrestrial integrated networks with non-orthogonal multiple access. IEEE J. Sel. Top. Signal Process. 13(3), 657-670 (2019)

38. Z. Lin, M. Lin, J. Ouyang, W. Zhu, A. D. Panagopoulos, M. Alouini, Robust secure beamforming for multibeam satellite communication systems. IEEE Trans. on Veh. Technol. 68(6), 6202-6206 (2019)

39. M. Ashraphijuo, V. Aggarwal, X. Wang, On the capacity regions of two-way diamond channels. IEEE Trans. Inf. Theory. 61(11), 6060-6090 (2015)

40. W. Kang, S. Ulukus, Capacity of a class of diamond channels. IEEE Trans. Inf. Theory. 57(8), 4955-4960 (2011)

41. Q. Wang, P. Fan, M. R. Mckay, K. B. Letaief, On the position selection of relays in diamond relay networks. IEEE Trans. Commun. 59(9), 2515-2527 (2011)

42. M. O. Hasna, M.-S. Alouini, in Proc. 2002 IEEE VTC. Performance analysis of two-hop relayed transmissions over Rayleigh fading channels. https://doi. org/10.1109/vetecf.2002.1040567

43. Y. Zeng, R. Zhang, Optimized training design for wireless energy transfer IEEE Trans. Commun. 63(2), 536-550 (2015)

44. T. Cui, F. Gao, T. Ho, A. Nallanathan, Distributed space-time coding for two-way wireless relay networks. IEEE Trans. Signal Proces. 57(2), 658-671 (2009)

45. J. Y. Ryu, J. Lee, T. Q. S. Quek, Trust degree based beamforming for MISO cooperative communication system. IEEE Commun. Lett. 19(11), 1957-1960 (2015)

46. J. Lindblom, E. Karipidis, E. G. Larsson, Efficient computation of pareto optimal beamforming vectors for the MISO interference channel with successive interference cancellation. IEEE Trans. Signal Proces. 61(19), 4782-4795 (2013)

47. C. Zhong, X. Chen, Z. Zhang, G. K. Karagiannidis, Wireless-powered communications: performance analysis and optimization. IEEE Trans. Commun. 63(12), 5178-5190 (2015)

48. R. Corless, G. Gonnet, D. Hare, D. Jeffrey, D. Knuth, in Advances in Computational Mathematics. On the LambertWfunction (Springer-Verlag, Berlin, 1996), p. 329C359

49. E. Bjornson, L. Sanguinetti, J. Hoydis, M. Debbah, Optimal design of energy-efficient multi-user MIMO systems: is massive MIMO the answer? IEEE Trans. Wirel. Commun. 14(6), 3059-3075 (2015)

\section{Publisher's Note}

Springer Nature remains neutral with regard to jurisdictional claims in published maps and institutional affiliations.

\section{Submit your manuscript to a SpringerOpen ${ }^{\circ}$ journal and benefit from:}

- Convenient online submission

- Rigorous peer review

- Open access: articles freely available online

- High visibility within the field

- Retaining the copyright to your article

Submit your next manuscript at $\gg$ springeropen.com 